\title{
Silylstannylation of Highly Functionalized Acetylenes. Synthesis of Precursors for Annulations via Radical or Heck Reactions
}

\author{
Sandeep Apte, Branko Radetich, Seunghoon Shin and T. V. RajanBabu* \\ Department of Chemistry, $100 \mathrm{~W} .18^{\text {th }}$ Ave., The Ohio State University, Columbus OH 43210
}

\section{SUPPORTING INFORMATION}

\section{Table of Contents}

$\begin{array}{ll}\text { Materials and Methods } & \text { S-3 }\end{array}$

Synthesis of $\mathbf{1 b} \quad$ S-3

Silylstannylation of $\mathbf{1 a}$ with $\mathrm{Me}_{3} \mathrm{SiSnBu}_{3} \quad \mathrm{~S}-3$

Silylstannylation of $\mathbf{1 b}$ with $\mathrm{Me}_{3} \mathrm{SiSnBu}_{3} \quad$ S-4

Reaction of 5a with iodine $\quad$ S-4

Reaction of $\mathbf{5 b}$ with iodine $\quad S-5$

Radical cyclization of $\mathbf{6 b}$ to $\mathbf{2 1 b} \quad \mathrm{S}-5$

Synthesis of $7 \quad$ S-5

Silylstannylation of 7 and conversion of the product to bicyclic b-lactam $23 \quad$ S-6

Silylstannylation of $9 \quad$ S-7

$\begin{array}{ll}\text { Synthesis of } \mathbf{1 1} & \mathrm{S}-7\end{array}$

Silylstannylation of $\mathbf{1 1} \quad$ S-8

Synthesis of $\mathbf{1 3} \quad$ S-8

Silylstannylation of $\mathbf{1 3} \quad$ S-10

$\begin{array}{ll}\text { Silylstannylation of substrate with unprotected alcohol } & \text { S-10 }\end{array}$

Silylstannylation of $\mathbf{1 5}$ and iodination of adduct (Synthesis of 29) S-11

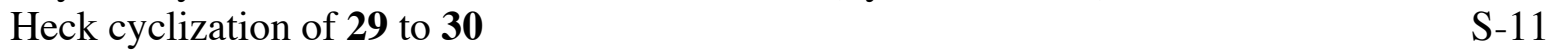

Synthesis of $(2 E, 4 E)$-2,4-hexadienyl 2-propynyl ether (34, entry 7, Table 1) S-12

Silylstannylation of $(2 E, 4 E)$-2,4-hexadienyl 2-propynyl ether 34 (entry 7, Table 1) S-12

Iodination of $\mathbf{3 5}\left(\mathrm{X}=\mathrm{SnBu}_{3}\right.$, entry 7, Table 1). $\quad \mathrm{S}-12$

Synthesis of 17a and 17b $\quad S-13$

Silylstannylation of 17a and iodination of 18a $\quad$ S-14

Radical cyclization of $\mathbf{2 4} \quad$ S-14

Iodination of 18b, Preparation of $\mathbf{2 6} \quad \mathrm{S}-15$

Heck cyclization of $\mathbf{2 6} \quad$ S-15

Acid-catalyzed isomerization of $\mathbf{2 7}$ to $\mathbf{2 8} \quad S-16$

Epoxide-mediated cyclizations $\quad$ S-16

$\begin{array}{ll}\text { Synthesis of 19a } & \text { S-16 }\end{array}$

Silylstannylation of 19a $\quad$ S-17

Titanocene monochloride-mediated cyclization of 20a $\quad$ S-17

$\begin{array}{lr}\text { Silylstannylation of } \mathbf{1 9 b} & \mathrm{S}-18\end{array}$

Titanocene monochloride-mediated cyclization of 20b $\quad$ S-18

Examples from Table 1, Supporting Information

$\begin{array}{lr}\text { (not included in Table } 1 \text { of printed version) } & \text { S-19 }\end{array}$

$\begin{array}{lr}\text { Silylstannylation of } \mathbf{3 2} & \text { S-19 }\end{array}$ 
Table 1. Complete list of substrates for Silylstannylation with reaction conditions

${ }^{1} \mathrm{H},{ }^{13} \mathrm{C}$ NMR of 4

${ }^{1} \mathrm{H},{ }^{13} \mathrm{C}$ NMR of $\mathbf{5 a}$

${ }^{1} \mathrm{H},{ }^{13} \mathrm{C}$ NMR of $\mathbf{5 b}$

${ }^{1} \mathrm{H},{ }^{13} \mathrm{C}$ NMR of $\mathbf{6 a}$

${ }^{1} \mathrm{H},{ }^{13} \mathrm{C}$ NMR of $\mathbf{6 b}$

${ }^{1} \mathrm{H},{ }^{13} \mathrm{C}$ NMR of 7

${ }^{1} \mathrm{H},{ }^{13} \mathrm{C}$ NMR of 8

${ }^{1} \mathrm{H},{ }^{13} \mathrm{C}$ NMR of 22

${ }^{1} \mathrm{H},{ }^{13} \mathrm{C}$ NMR of 23

${ }^{1} \mathrm{H}, \mathrm{nOe}{ }^{13} \mathrm{C}$ NMR of $\mathbf{1 0}$

${ }^{1} \mathrm{H}, \mathrm{COSY},{ }^{13} \mathrm{C}$ NMR, GC of $\mathbf{1 1}$

${ }^{1} \mathrm{H}, \mathrm{COSY},{ }^{13} \mathrm{C}$ NMR of $\mathbf{1 2}$

${ }^{1} \mathrm{H}, \mathrm{nOe},{ }^{13} \mathrm{C}$ NMR of $\mathbf{1 4}$

${ }^{1} \mathrm{H},{ }^{13} \mathrm{C}$ NMR of $\mathbf{1 6}$

${ }^{1} \mathrm{H},{ }^{13} \mathrm{C}$ NMR of 29

${ }^{1} \mathrm{H},{ }^{13} \mathrm{C}$ NMR of $\mathbf{3 0}$

${ }^{1} \mathrm{H}, \mathrm{NMR}$ of $\mathbf{1 7 a}$

${ }^{1} \mathrm{H}, \mathrm{NMR}$ of $\mathbf{1 7} \mathbf{b}$

${ }^{1} \mathrm{H},{ }^{13} \mathrm{C}$ NMR of $\mathbf{1 8 a}$

${ }^{1} \mathrm{H},{ }^{13} \mathrm{C}$ NMR of 24

${ }^{1} \mathrm{H},{ }^{13} \mathrm{C}$ NMR of 25

${ }^{1} \mathrm{H},{ }^{13} \mathrm{C}$ NMR of $\mathbf{1 8 b}$

${ }^{1} \mathrm{H},{ }^{13} \mathrm{C}$ NMR of 26

${ }^{1} \mathrm{H},{ }^{13} \mathrm{C}$ NMR of 27

${ }^{1} \mathrm{H},{ }^{13} \mathrm{C}$ NMR of 28

${ }^{1} \mathrm{H},{ }^{13} \mathrm{C}$ NMR, CG of 19a

${ }^{1} \mathrm{H},{ }^{13} \mathrm{C},{ }^{119} \mathrm{Sn}$ NMR of 20a

${ }^{1} \mathrm{H}, \mathrm{NOESY},{ }^{13} \mathrm{C}$ NMR, GC of 31a

${ }^{1} \mathrm{H},{ }^{13} \mathrm{C},{ }^{119} \mathrm{Sn}$ NMR of $\mathbf{2 0 b}$

${ }^{1} \mathrm{H}, \mathrm{NOESY},{ }^{13} \mathrm{C}$ NMR of $\mathbf{3 1 b}$-major

${ }^{1} \mathrm{H},{ }^{13} \mathrm{C}$ NMR of 31b-minor

Expanded ${ }^{1} \mathrm{H}$, NMR of 31b-major+ minor

GC of 31b-major+ minor

${ }^{1} \mathrm{H},{ }^{13} \mathrm{C}$ NMR of $\mathbf{3 3}$ (structure in SI-Table 1, entry 11)

${ }^{1} \mathrm{H},{ }^{13} \mathrm{C}$ NMR of 35 (structure in SI-Table 1, entry 7)

${ }^{1} \mathrm{H},{ }^{13} \mathrm{C}$ NMR of $\mathbf{3 5}+\mathrm{I}_{2}$ (structure in SI-Table 1, entry 7)

${ }^{1} \mathrm{H},{ }^{13} \mathrm{C}$ NMR of 37 (structure in SI-Table 1, entry 12)

${ }^{1} \mathrm{H},{ }^{13} \mathrm{C}$ NMR of $\mathbf{3 8}$ (structure in SI-Table 1, entry 12)

${ }^{1} \mathrm{H}$, nOe, ${ }^{13} \mathrm{C}$ NMR of product, entry 13 SI-Table 1

${ }^{1} \mathrm{H},{ }^{13} \mathrm{C}$ NMR of product, entry 14 SI-Table 1

${ }^{1} \mathrm{H},{ }^{13} \mathrm{C}$ NMR of product, entry 15 SI-Table 1

${ }^{1} \mathrm{H},{ }^{13} \mathrm{C}$ NMR of product, entry 16 SI-Table 1 
Materials and Methods. All reactions were carried out using dry, freshly distilled solvents. Diethyl ether $\left(\mathrm{Et}_{2} \mathrm{O}\right)$ and tetrahydrofuran (THF) were distilled from sodium/benzophenone, and dichloromethane $\left(\mathrm{CH}_{2} \mathrm{Cl}_{2}\right)$ from calcium hydride. Solvents were stored over molecular sieves. All reactions were monitored by ${ }^{1} \mathrm{H}$ NMR spectroscopy (in $\mathrm{C}_{6} \mathrm{D}_{6}$ ) or thin layer chromatography (TLC) using $0.25-\mathrm{mm}$ E. Merck precoated silica gel $60 \mathrm{~F}_{254}$ plates. Flash chromatography was performed with the indicated solvents and silica gel of particle size $0.040-0.063 \mathrm{~mm}$. Yields refer to chromatographically and spectroscopically pure compounds unless otherwise indicated. Melting points were obtained on a Thomas-Hoover apparatus. High resolution mass spectra were obtained at the mass spectrometry facility in the department of chemistry, the Ohio State University. Elemental analyses was done by Atlantic Microlab Inc., GA.

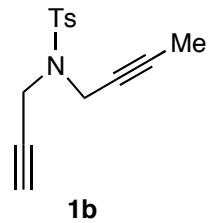

Synthesis of $1 \mathbf{b}$. To a solution of dipropargyl tosylamine (284 $\mathrm{mg}, 1.15 \mathrm{mmol})$ in $5 \mathrm{~mL}$ of THF at $-78{ }^{\circ} \mathrm{C}$ was added $n$-BuLi $(0.92 \mathrm{~mL}$ of $2.5 \mathrm{M}$ in Hex, $2.30 \mathrm{mmol})$ and the reaction mixture was stirred for $30 \mathrm{~min}$. To the mixture was added $\mathrm{CuI}(219 \mathrm{mg}, 1.15$ mmol), followed by MeI $(0.071 \mathrm{~mL}, 1.15 \mathrm{mmol})$. The mixture was stirred at $-78{ }^{\circ} \mathrm{C}$ for $1 \mathrm{~h}$, then was allowed to warm to rt over $3 \mathrm{~h}$ while stirring. The reaction was quenched by addition of $30 \mathrm{~mL}$ of water. The aqueous layer was extracted with $\mathrm{CH}_{2} \mathrm{Cl}_{2}(50 \mathrm{~mL} \times 3)$. When the emulsion formed, additional brine $(20 \mathrm{~mL})$ was added. The combined organic layer was dried $\left(\mathrm{MgSO}_{4}\right)$, evaporated, and the residue was subjected to flash chromatography $(\mathrm{EtOAc} / \mathrm{Hex}=1 / 5)$ to get $164 \mathrm{mg}(55 \%)$ of a pale yellow liquid. 1b: ${ }^{1} \mathrm{H}$ NMR (500 MHz, $\left.\mathrm{CDCl}_{3}\right): \square 1.64(\mathrm{t}, \mathrm{J}=2.3 \mathrm{~Hz}, 3 \mathrm{H}), 2.13(\mathrm{t}, \mathrm{J}=2.5 \mathrm{~Hz}, \mathrm{H})$, 2.42 (s, 3H), 4.09 (q, J = 2.2 Hz, 2H), 4.13 (d, J = 2.3 Hz, 2H), 7.29 (d, J = 8.0 Hz, 2H), 7.71 (d, J = 8.3 $\mathrm{Hz}, 2 \mathrm{H}) ;{ }^{13} \mathrm{C}\left(125 \mathrm{MHz}, \mathrm{CDCl}_{3}\right)$ : $\mathrm{Q} 3.8,21.9,36.5,37.1,71.7,74.1,77.0,82.4,128.4,129.8,135.9$, 144.1 .

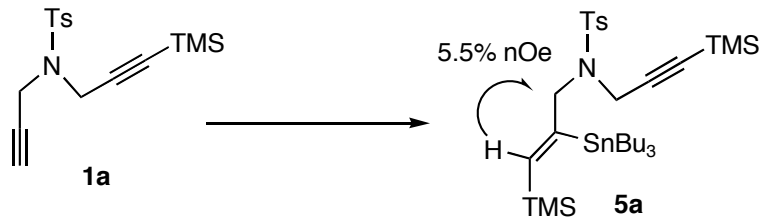

Silylstannylation of 1a. To a solution of $\mathrm{Pd}_{2}(\mathrm{dba})_{3}(0.9$ $\mathrm{mg}, 0.002 \mathrm{mmol} \mathrm{Pd})$ and a phosphine ligand $(0.004$ $\mathrm{mmol})$ in $1 \mathrm{~mL}$ of $\mathrm{C}_{6} \mathrm{D}_{6}$ was added $\mathrm{Bu}_{3} \mathrm{Sn}-\mathrm{SiMe}_{3}(40.0$ $\mathrm{mg}, 0.110 \mathrm{mmol})$ followed by the diyne $1 \mathrm{a}(31.9 \mathrm{mg}, 0.100 \mathrm{mmol})$. The reactions using various ligands $\left(\mathrm{PCy}_{3}, \mathrm{PBu}_{3}{ }_{3}, \mathrm{PPh}_{3}\right.$, and $\left.\mathrm{P}(\mathrm{OPh})_{3}\right)$ were monitored by ${ }^{1} \mathrm{H}$ NMR. In a reaction using $\mathrm{PCy}_{3}(1.1$ $\mathrm{mg}$ ), the reaction was complete in $16 \mathrm{~h}$ at $\mathrm{rt}\left({ }^{1} \mathrm{H} \mathrm{NMR}\right)$. The solvent was removed from the reaction mixture and the residue was subjected to flash chromatography (EtOAc/Hex $=1 / 16)$ to get $60.0 \mathrm{mg}(88$ \%) of 5a as off-white crystal (mp 60-62 $\left.{ }^{\circ} \mathrm{C}\right) .5 \mathbf{a}:{ }^{1} \mathrm{H}$ NMR $\left(500 \mathrm{MHz}, \mathrm{CDCl}_{3}\right)$ : $\square-0.05$ (s, 9H), 0.10 (s, 
9H), $\mathrm{SnBu}_{3}$ peaks are omitted, $2.42(\mathrm{~s}, 3 \mathrm{H}), 3.97(\mathrm{~s}, 2 \mathrm{H}), 3.99(\mathrm{~m}, 2 \mathrm{H}), 6.67\left(\mathrm{~s}, \mathrm{~J}_{\mathrm{Sn}-\mathrm{H}}=158 \mathrm{~Hz}, \mathrm{H}\right)$, $7.27(\mathrm{~d}, \mathrm{~J}=8.3 \mathrm{~Hz}, 2 \mathrm{H}), 7.71(\mathrm{~d}, \mathrm{~J}=8.3 \mathrm{~Hz}, 2 \mathrm{H}) ;{ }^{13} \mathrm{C} \mathrm{NMR}\left(125 \mathrm{MHz}, \mathrm{CDCl}_{3}\right): \square-0.24,0.27,10.5$, 14.0, 21.2 28.0, 29.7, 37.0, 60.9, 90.9, 98.5, 128.6, 129.5, 136.8, 142.9, 149.1, 157.8; HRMS calcd for $[\mathrm{M}+\mathrm{Na}]^{+} 706.2563$, found 706.2573; nOe difference spectra confirmed the double bond geometry shown above.

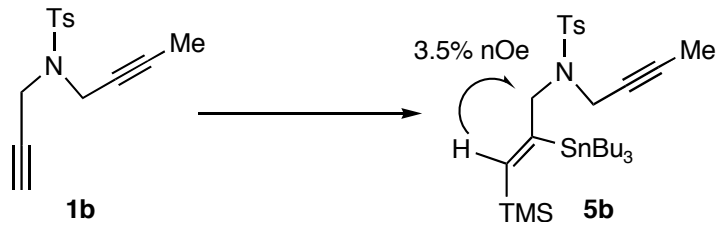

Silylstannylation of $\mathbf{1 b}$. To a solution of $\mathrm{Pd}_{2}(\mathrm{dba})_{3}(1.8$ $\mathrm{mg}, 0.004 \mathrm{mmol} \mathrm{Pd})$ and a phosphine ligand $(0.008 \mathrm{mmol})$ in $1 \mathrm{~mL}$ of $\mathrm{C}_{6} \mathrm{D}_{6}$ was added $\mathrm{Bu}_{3} \mathrm{Sn}_{-} \mathrm{SiMe}_{3}(80 \mathrm{mg}, 0.22$ mmol) followed by diyne $1(\mathrm{R}=\mathrm{Me}, 52.3 \mathrm{mg}, 0.200 \mathrm{mmol})$. The reactions using various ligands $\left(\mathrm{PCy}_{3}, \mathrm{PBu}_{3}{ }_{3}, \mathrm{PPh}_{3}\right.$, and $\left.\mathrm{P}(\mathrm{OPh})_{3}\right)$ were monitored by ${ }^{1} \mathrm{H}$ NMR. In a reaction using $\mathrm{P}(\mathrm{OPh})_{3}(2.5 \mathrm{mg})$, the reaction was complete in $17 \mathrm{~h}$ at $60{ }^{\circ} \mathrm{C}\left({ }^{1} \mathrm{H} \mathrm{NMR}\right)$. The solvent was removed from the reaction mixture and the residue was subjected to flash chromatography (EtOAc/Hex =1/12) to get $83.6 \mathrm{mg}(67$ \%) of $\mathbf{5 b}$ as a colorless oil. 5b: ${ }^{1} \mathrm{H}$ NMR (400 MHz, $\left.\mathrm{CDCl}_{3}\right)$ : $\square 0.13$ (s, 9H), $\mathrm{SnBu}_{3}$ peaks are omitted, $1.51(\mathrm{t}, \mathrm{J}=2.3 \mathrm{~Hz}, 3 \mathrm{H}, \mathrm{C} \equiv \mathrm{CMe}), 2.45(\mathrm{~s}, 3 \mathrm{H}), 3.96\left(\mathrm{~d}, \mathrm{~J}=2.1 \mathrm{~Hz}, 2 \mathrm{H}\right.$, propargyl $\left.\mathrm{CH}_{2}\right), 3.99$ (s br, 2H, allyl $\left.\mathrm{CH}_{2}\right), 6.66\left(\mathrm{~s}, J_{\mathrm{Sn}-\mathrm{H}}=162 \mathrm{~Hz}, \mathrm{H}\right), 7.32(\mathrm{~d}, \mathrm{~J}=8.1 \mathrm{~Hz}, 2 \mathrm{H}), 7.76(\mathrm{~d}, \mathrm{~J}=8.1 \mathrm{~Hz}, 2 \mathrm{H}) ;{ }^{13} \mathrm{C}$ NMR $\left(100 \mathrm{MHz}_{\mathrm{CDCl}}\right)$ : $\square-0.1,2.9,10.9,13.5,21.3,27.2,29.0,36.1,58.6,71.2,81.4,127.9,128.9,136.0$, 142.9, 146.0, 156.0; HRMS calcd for $[\mathrm{M}+\mathrm{Na}]^{+}$648.2324, found 648.2336; nOe difference spectra confirmed the double bond geometry shown above.

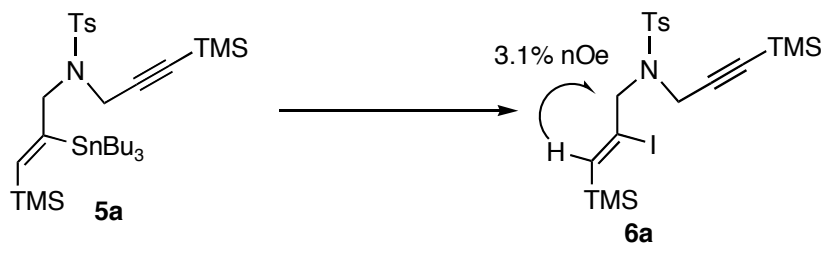

Reaction of $5 \mathbf{a}$ with iodine. To a solution of $5 \mathbf{a}$ (34.2 $\mathrm{mg}, 0.0500 \mathrm{mmol})$ in $\mathrm{CH}_{2} \mathrm{Cl}_{2}(2 \mathrm{~mL})$ at $\mathrm{rt}$ was added $\mathrm{I}_{2}(15 \mathrm{mg}, 0.060 \mathrm{mmol})$ and the mixture was stirred at $\mathrm{rt}$ for $20 \mathrm{~min}$. The solvent was evaporated and the residue was subjected to flash chromatography (EtOAc/Hex $=1 / 16)$ to get $25.5 \mathrm{mg}(98 \%)$ of 6a as a white crystalline solid (mp 101-102 $\left.{ }^{\circ} \mathrm{C}\right)$. 6a: ${ }^{1} \mathrm{H}$ NMR (400 MHz, $\left.\mathrm{CDCl}_{3}\right)$ : $\square 0.00$ (s, 9H), 0.20 (s, 9H), 2.42 (s, 3H), 4.08 (s, 4H, allyl and propargyl $\left.\mathrm{CH}_{2}\right), 6.75(\mathrm{~s}, \mathrm{H}), 7.29(\mathrm{~d}, \mathrm{~J}=8.0 \mathrm{~Hz}, 2 \mathrm{H}), 7.72$ $(\mathrm{d}, \mathrm{J}=8.0 \mathrm{~Hz}, 2 \mathrm{H}) ;{ }^{13} \mathrm{C} \mathrm{NMR}\left(125 \mathrm{MHz}, \mathrm{CDCl}_{3}\right)$ : $\square-1.4,-0.5,21.4,37.0,61.1,91.3,97.2,113.2$, 127.6, 129.4, 136.0, 140.2, 143.5; HRMS calcd for $[\mathrm{M}+\mathrm{Na}]^{+}$542.0473, found 542.0472; nOe difference spectra confirmed the double bond geometry shown above. 


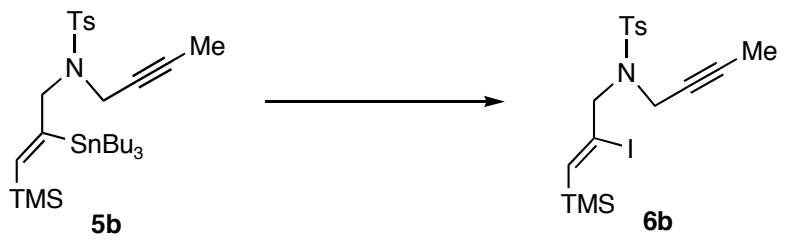

Reaction of $\mathbf{5 b}$ with iodine. To a solution of $\mathbf{5 b}(29.4$ $\mathrm{mg}, 0.0471 \mathrm{mmol})$ in $\mathrm{CH}_{2} \mathrm{Cl}_{2}(2 \mathrm{~mL})$ at rt was added $\mathrm{I}_{2}$ (24 mg, $0.094 \mathrm{mmol}$ ) and the mixture was stirred at $\mathrm{rt}$ for $20 \mathrm{~min}$. The solvent was evaporated and the residue was subjected to flash chromatography $(\mathrm{EtOAc} / \mathrm{Hex}=1 / 9)$ to get $19.4 \mathrm{mg}(90 \%)$ of the product. $6 \mathbf{b}$ : ${ }^{1} \mathrm{H}$ NMR (500 MHz, $\left.\mathrm{CDCl}_{3}\right) \square 0.19(\mathrm{~s}, 9 \mathrm{H}), 1.56$ (t, J = 2.3 Hz, 3H), 2.42 (s, 3H), 4.01 (q, J = 2.3 Hz, 2H, propargyl $\left.\mathrm{CH}_{2}\right), 4.08\left(\mathrm{~d}, \mathrm{~J}=1.1 \mathrm{~Hz}, 2 \mathrm{H}\right.$, allyl $\left.\mathrm{CH}_{2}\right), 6.72(\mathrm{~s}, \mathrm{H}), 7.29(\mathrm{~d}, \mathrm{~J}=8.1 \mathrm{~Hz}, 2 \mathrm{H}), 7.73(\mathrm{~d}, \mathrm{~J}$ $=8.3 \mathrm{~Hz}, 2 \mathrm{H}) ;{ }^{13} \mathrm{C}$ NMR $\left(125 \mathrm{MHz}, \mathrm{CDCl}_{3}\right) \square-0.8,3.7,21.9,37.4,61.7,71.9,82.3,114.3,128.2$, 129.7, 136.8, 139.6, 143.9; HRMS calcd for $[\mathrm{M}+\mathrm{Na}]^{+} 484.0234$, found 484.0212; nOe difference spectra confirmed the double bond geometry shown above.

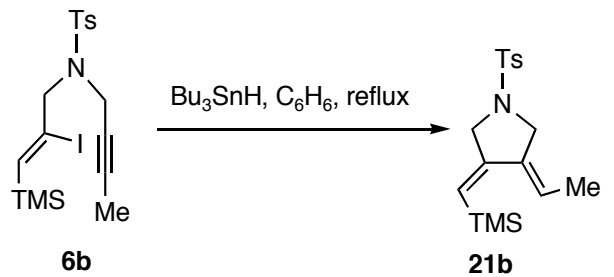

Radical cyclization of $\mathbf{6 b}$ to $\mathbf{2 1 b}$. To a refluxing solution of
starting iodide $\mathbf{6 b}(14.8 \mathrm{mg}, 0.0321 \mathrm{mmol})$ in $3 \mathrm{~mL}$ of benzene
was added via syringe pump a solution of $n-\mathrm{Bu} 3 \mathrm{SnH}(19.9 \mathrm{mg}$,
$0.0685 \mathrm{mmol}, 2 \mathrm{eq})$ and AIBN $(1.7 \mathrm{mg}, 0.010 \mathrm{mmol}, 0.3 \mathrm{eq})$ in 1 $\mathrm{mL}$ of benzene, over $3 \mathrm{~h}$. After $3.5 \mathrm{~h}$, the solvent was removed from the reaction mixture and the residue was purified by chromatography to get $7.0 \mathrm{mg}$ of a mixture of $\mathbf{2 1 b}$ and starting material (2:1 molar ratio). The yield of the product was estimated as $48 \%$. 21b: ${ }^{1} \mathrm{H}$ NMR $\left(500 \mathrm{MHz}, \mathrm{CDCl}_{3}\right)$ : 0.10 (s, 9 H, TMS), 1.66 (d, J = 7.2 Hz, $3 \mathrm{H}, \mathrm{C} \equiv \mathrm{C}-\mathrm{CH}_{3}$ ), 2.43 (s, 3H), 3.92 (t, br, J = 2.2 Hz, 2H, $\mathrm{CH}_{2} \mathrm{C}=\mathrm{CHMe}$ ), $3.97\left(\mathrm{~d}, \mathrm{~J}=2.5 \mathrm{~Hz}, 2 \mathrm{H}, \mathrm{CH}_{2} \mathrm{C}=\mathrm{CHSi}\right), 5.80$ (t, br, J = 2.3 Hz, H, C=CHTMS), 5.90 $(\mathrm{m}, \mathrm{H}, \mathrm{C}=\mathrm{C} H \mathrm{Me}), 7.33(\mathrm{~d}, \mathrm{~J}=8.2 \mathrm{~Hz}, 2 \mathrm{H}, \operatorname{Ar}-H), 7.72(\mathrm{~d}, \mathrm{~J}=8.2 \mathrm{~Hz}, 2 \mathrm{H}, \operatorname{Ar}-H) ;{ }^{13} \mathrm{C}(125 \mathrm{MHz}$, $\left.\mathrm{CDCl}_{3}\right):-0.2,22.0,31.3,50.6,53.4,116.8,117.6,128.2,130.1,133.2,136.0,144.1,148.7 ; \mathrm{nOe}$ experiments confirmed the double bond geometry.

Synthesis of 7. The $\beta$-lactam ${ }^{1}(0.40 \mathrm{~g}, 1.6 \mathrm{mmol})$ was dissolved in $2 \mathrm{~mL}$ of THF and was added dropwise to a cooled slurry $\left(0^{\circ} \mathrm{C}\right.$, ice/acetone $)$ of $\mathrm{NaH}(0.04 \mathrm{~g}, 1.8 \mathrm{mmol}, 1.1$ equiv. $)$ in $2 \mathrm{~mL}$ of THF. After 1 hour of stirring, propargyl bromide $(0.25 \mathrm{~g}, 1.8 \mathrm{mmol}, 1.1$ equiv.) was added dropwise to the solution. After one hour the reaction was complete with disappearance of the starting material, and appearance of two spots on TLC (7/3 hexane/ethyl acetate). The less polar spot, by far the major one, proved to be the product. Reaction was stopped and the salts were filtered off on a celite packed sintered filter. Purification was performed by flash column chromatography (hexane/ethyl acetate,

(1) We thank Professor D. Hart for a sample of the starting G-lactam. See ref. 5 in the paper. 
7/3), to yield $0.32 \mathrm{~g}(72 \%)$, of a thick yellowish oil. ${ }^{1} \mathrm{H} \mathrm{NMR} \square\left(\mathrm{CDCl}_{3}\right): 1.07(\mathrm{~d}, \mathrm{~J}=6.6 \mathrm{~Hz}, 3 \mathrm{H}$, $\left.\left.\mathrm{CHCH}_{3}\right), 1.12(\mathrm{~d}, \mathrm{~J}=10.3 \mathrm{~Hz}, 5.2 \mathrm{~Hz}, 1 \mathrm{H}, \mathrm{CHC}=\mathrm{O}), 3.80(\mathrm{~s}, 3 \mathrm{H}, \mathrm{Ar}-\mathrm{OCH})_{3}\right), 3.82(\mathrm{dd}, \mathrm{J}=16.8 \mathrm{~Hz}, 2.5$ Hz, 1H, NCH-propargyl), 4.32 (dd, J = 17.7 Hz, $2.5 \mathrm{~Hz}, 1 \mathrm{H}, \mathrm{NCH}$-propargyl), 4.59 (d, J = 5.19 Hz, 1H, NCH-Ar), 6.84 (d, J = 8.9 Hz, 2H, Ar- $H$ ), 7.33 (d, J = 8.9 Hz, 2H, Ar- $H)$; ${ }^{13} \mathrm{C} \mathrm{NMR} \square\left(\mathrm{CDCl}_{3}\right)$ : 19.9, 21.5, 27.0, 29.3, 45.9, 55.2, 61.7, 72.2, 76.8, 81.5, 87.8, 113.9, 114.0, 133.1, 159.9, 168.2; HRMS 281.1416. Calcd for $\mathrm{C}_{18} \mathrm{H}_{19} \mathrm{O}_{2} 281.1416$.

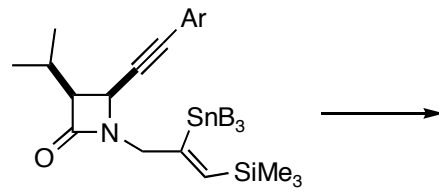

8

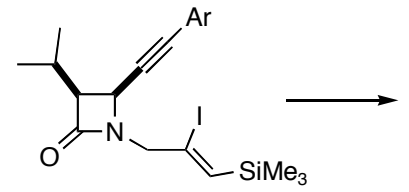

22

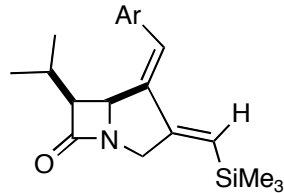

23

Silylstannylation of 7 and conversion of the product to bicyclic $\square$-lactam 23. Silylstannylation of 7 . The $\beta$ lactam $7(0.10 \mathrm{~g}, 0.4 \mathrm{mmol})$ was dissolved in $2 \mathrm{~mL}$ of benzene and placed in a three neck $25 \mathrm{~mL}$ round bottom flask. To the solution was added $\mathrm{Bu}_{3} \mathrm{SnSiMe}_{3}(0.14 \mathrm{~g}, 0.4 \mathrm{mmol}), 1$ equiv.) followed by $\mathrm{Pd}\left(\mathrm{PPh}_{3}\right)_{4}(21 \mathrm{mg}, 0.018 \mathrm{mmol}, 0.05$ equiv $)$. Final volume after the transfer of all the reagents was approximately $5 \mathrm{~mL}$. The mixture was heated for six hours, under reflux conditions. After this period a major spot evolved on TLC ( $\mathrm{rf}=0.45$, hexane/ethyl acetate 7:3), and even after additional day of refluxing there was no obvious change in the appearance of the TLC plate. Solution was concentrated, and crude was subjected to flash chromatography (hexane/ethyl acetate 7:3). 8: ${ }^{1} \mathrm{H} \mathrm{NMR} \square\left(\mathrm{CDCl}_{3}\right)$ : 0.11 (s, 9H, $\mathrm{Si}\left(\mathrm{CH}_{3}\right)_{3}, 0.85-1.02$ (m, 14H, SnCH's), 1.08 (d, J = 6.5 Hz, 3H, $\left.\mathrm{CH}_{3} \mathrm{CHRCH}_{3}\right), 1.22$ (d, J $\left.=6.7 \mathrm{~Hz}, 3 \mathrm{H}, \mathrm{CH}_{3} \mathrm{CHRCH}_{3}\right), 1.25-1.53\left(\mathrm{~m}, 13 \mathrm{H}, \mathrm{SnCH}\right.$ 's), 2.19-2.32 (m, 1H, $\left.\mathrm{CH}_{3} \mathrm{CHRCH}_{3}\right), 3.04$ (dd, $\mathrm{J}=10.4 \mathrm{~Hz}, 5.1 \mathrm{~Hz}, 1 \mathrm{H}, \mathrm{CHC}=\mathrm{O}), 3.74\left(\mathrm{~d}, \mathrm{~J}=16.7 \mathrm{hz}, 1 \mathrm{H}, \mathrm{R}_{2} \mathrm{~N}-\mathrm{CHCSnBu} 3=\mathrm{CHSi}\left(\mathrm{CH}_{3}\right)_{3}, 3.81(\mathrm{~s}\right.$, $\left.3 \mathrm{H}, \mathrm{Ar}-\mathrm{OCH}_{3}\right), 4.30\left(\mathrm{dd}, \mathrm{J}=15.8 \mathrm{~Hz}, 1.7 \mathrm{~Hz}, \mathrm{H}, \mathrm{R}_{2} \mathrm{~N}-\mathrm{CHCSnBu} 3=\mathrm{CHSi}_{3}\left(\mathrm{CH}_{3}\right)_{3}, 4.38(\mathrm{~d}, \mathrm{~J}=5.1 \mathrm{~Hz}\right.$, 1H N-CH-propargyl), 6.54 (s, 1H, $-\mathrm{CSnBu}_{3}=\mathrm{CHSi}\left(\mathrm{CH}_{3}\right)_{3}, 6.84$ (d, J = 8.7, Hz, 2H, aromatic), 7.33 (d, $\mathrm{J}=8.8 \mathrm{~Hz}, 1 \mathrm{H}$, aromatic); ${ }^{13} \mathrm{C} \mathrm{NMR} \square\left(\mathrm{CDCl}_{3}\right): 0.11(\mathrm{q}), 10.8(\mathrm{t}), 13.5(\mathrm{q}), 20.0$ (q), $21.6(\mathrm{q}), 27.3$ (q), $29.0(\mathrm{t}), 46.2(\mathrm{t}), 52.3(\mathrm{t}), 55.2(\mathrm{q}), 60.8(\mathrm{~d}), 82.0(\mathrm{~s}), 87.8(\mathrm{~s}), 113.9(\mathrm{~d}), 114.2(\mathrm{~s}), 133.0(\mathrm{~d}), 145.5(\mathrm{~d})$, 156.0 (s), 169.3 (s); Anal. Calcd for $\mathrm{C}_{33} \mathrm{H}_{55} \mathrm{O}_{2} \mathrm{NSiSn} \mathrm{C}, 61.37$ H, 8.59, Found: C, 61.48 H, 8.64.

Iodination of 8. The starting silylstannane $8(0.27 \mathrm{~g}, 0.61 \mathrm{mmol})$ in $\mathrm{CH}_{2} \mathrm{Cl}_{2}$ was titrated with $\mathrm{I}_{2}$ solution in $\mathrm{CH}_{2} \mathrm{Cl}_{2}$ at $0{ }^{\circ} \mathrm{C}$. After the titration was complete, the solution was concentrated and the crude product purified by flash chromatography to yield $0.27 \mathrm{~g}$ of iodide 22 (90\%).

Radical cyclization of iodide 22. The iodide was subjected to radical cyclization as follows: The iodide $22(0.10 \mathrm{~g}, 0.21 \mathrm{mmol})$ was dissolved in $11 \mathrm{~mL}$ of toluene $(0.02 \mathrm{M})$. The solution was placed in 
three-necked round bottom flask, fitted with a reflux condenser and heated at $90{ }^{\circ} \mathrm{C}$. To the refluxing solution, a solution of $\mathrm{Bu}_{3} \mathrm{SnH}(0.90 \mathrm{~g}, 0.3 \mathrm{mmol}, 1.5$ equiv.) and $\mathrm{AIBN}$ (7 mg, $0.04 \mathrm{mmol}, 0.2$ equiv.) in $4 \mathrm{~mL}$ of toluene was added slowly via syringe pump overnight. After the addition was complete, solvent was evaporated and the crude was purified via flash chromatography (hexane/ether $=9 / 1)$ to yield $45 \mathrm{mg}$ of 23 (61\%). Stereochemistry was confirmed by nOe studies. 23: ${ }^{1} \mathrm{H}$ NMR (400 MHz, $\left.\mathrm{CDCl}_{3}\right) \square 0.08$ (s, 9H, TMS), 0.87 (d, J =6.9 Hz, 3H, CHMe $), 1.05$ (d, J =6.6 Hz, 3H, CHMe $), 1.87-$ $1.92(\mathrm{~m}, \mathrm{H}, \mathrm{CHMe}$ ), $3.26(\mathrm{~s}, 3 \mathrm{H}, \mathrm{ArOMe}), 3.31$ (t, J = 5.5 Hz, H, CHCO), 3.39-4.64 (Abq, 口a = 3.41 ppm, $\square_{\mathrm{b}}=4.63$ ppm, $\left.\mathrm{J}=15.1 \mathrm{~Hz}, 2 \mathrm{H}, \mathrm{NCH}_{2}\right), 4.58-4.60(\mathrm{~m}, \mathrm{H}, \mathrm{NCH}), 6.11(\mathrm{~s}, \mathrm{H}, \mathrm{C}=\mathrm{CHTMS}), 6.74$ $(\mathrm{d}, \mathrm{J}=8.8 \mathrm{~Hz}, 2 \mathrm{H}, \mathrm{Ar}-H), 6.92(\mathrm{~s}, \mathrm{H}, \mathrm{C}=\mathrm{CHAr}), 7.14$ (d, J = 8.8 Hz, 2H, Ar- H); ${ }^{13} \mathrm{C} \mathrm{NMR}(100 \mathrm{MHz}$, $\left.\mathrm{CDCl}_{3}\right):-0.18(\mathrm{q}), 20.25(\mathrm{q}), 23.84(\mathrm{q}), 27.28(\mathrm{~d}), 50.78$ (t), $55.21(\mathrm{q}), 58.88(\mathrm{~d}), 63.62(\mathrm{~d}), 114.9(\mathrm{~d})$, 118.2 (d), 125.6 (d), 129.9 (s), 131.2 (d), 135.9 (s), 157.6 (s), 160.3 (s), 179.7 (s); Anal. Calcd for $\mathrm{C}_{21} \mathrm{H}_{29} \mathrm{NO}_{2} \mathrm{Si}: \mathrm{C}, 70.95, \mathrm{H}, 8.23$. Found: C, 70.85, H 8.23.

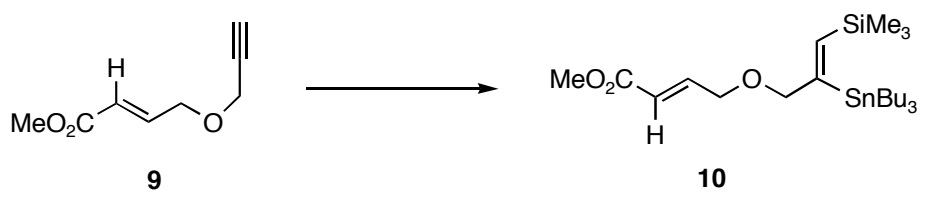

9

Silylstannylation of 9 . To a solution of $\mathrm{Pd}_{2}(\mathrm{dba})_{3}(1.8 \mathrm{mg}, 0.0020 \mathrm{mmol} \mathrm{Pd})$ and tris(o-tolyl)phosphine $(1.2 \mathrm{mg}, 0.0040 \mathrm{mmol})$ in 1 $\mathrm{mL}$ of $\mathrm{C}_{6} \mathrm{D}_{6}$ was added $\mathrm{Bu}_{3} \mathrm{Sn}_{-} \mathrm{SiMe}_{3}(80 \mathrm{mg}, 0.22 \mathrm{mmol})$ followed by eneyne $9(30.8 \mathrm{mg}, 0.200$ mmol). The reaction was monitored by ${ }^{1} \mathrm{H}$ NMR. After 4 days at $\mathrm{rt},{ }^{1} \mathrm{H}$ NMR indicated a good conversion into 10 and no starting material was left. 10: IR (neat): 2954, 2852, 1729, 1666, 1463, 1376, 1340, 1304, 1248, 1169, 1124, 1072, 1036, 965, $839 \mathrm{~cm}^{-1} ;{ }^{1} \mathrm{H}$ NMR (500 MHz, $\left.\mathrm{C}_{6} \mathrm{D}_{6}\right)$ : $\square 0.29$ (s, $9 \mathrm{H}), \mathrm{Bu}_{3} \mathrm{Sn}$ peaks are omitted, $3.49\left(\mathrm{~s}, 3 \mathrm{H}, \mathrm{CO}_{2} \mathrm{Me}\right), 3.80(\mathrm{dd}, \mathrm{J}=2.0,4.0 \mathrm{~Hz}, 2 \mathrm{H}$, $\left.\mathrm{OCH}_{2} \mathrm{CH}=\mathrm{CHCO}_{2} \mathrm{Me}\right), 4.09(\mathrm{~d}, \mathrm{~J}=1.6 \mathrm{~Hz}, 2 \mathrm{H}), 6.33\left(\mathrm{td}, \mathrm{J}=2.2,15.6 \mathrm{H}, \mathrm{H}, \mathrm{C}=\mathrm{CHCO}_{2} \mathrm{Me}\right), 6.77(\mathrm{~d}, \mathrm{~J}$ $\left.=1.4 \mathrm{~Hz},{ }^{3} J_{\mathrm{Sn}-\mathrm{H}}=160 \mathrm{~Hz}, \mathrm{H}, \mathrm{C}(\mathrm{Sn})=\mathrm{C}(\mathrm{Si}) H\right), 7.06\left(\mathrm{td}, \mathrm{J}=4.2,15.9 \mathrm{~Hz}, \mathrm{H}, \mathrm{RC} H=\mathrm{CHCO}_{2} \mathrm{Me}\right) ;{ }^{13} \mathrm{C}$ NMR (125 MHz, $\left.\mathrm{CDCl}_{3}\right)$ : $\square$ 0.5, 11.5, 14.0, 27.9, 29.6, 51.9, 68.8, 83.5, 121.2, 145.1, 145.4, 161.4, 167.2 (ester); HRMS calcd for $[\mathrm{M}+\mathrm{Na}]^{+}$541.2131, found 541.2149.

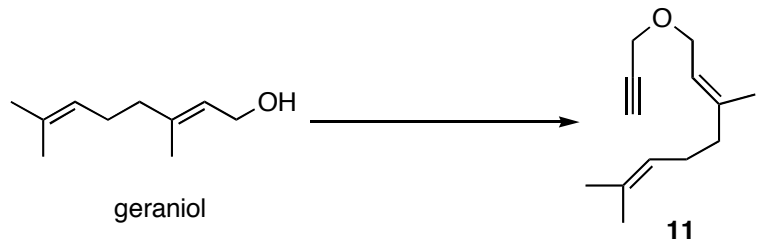

Synthesis of 11. To a solution of geraniol $(1.73 \mathrm{~mL}$, $10.0 \mathrm{mmol})$ in THF $(15 \mathrm{~mL})$ was added $\mathrm{KH}(441 \mathrm{mg}$, $11.0 \mathrm{mmol}$ ) portionwise at $\mathrm{rt}$. The reaction mixture was 
stirred for $2 \mathrm{~h}$ and propargyl bromide $(1.02 \mathrm{~mL}, 12.0 \mathrm{mmol})$ was added after cooling the mixture to 0 ${ }^{\circ} \mathrm{C}$. The resulting mixture was allowed to stir overnight. The reaction was quenched with addition of saturated aqueous solution of $\mathrm{NH}_{4} \mathrm{Cl}(50 \mathrm{~mL})$ and mixture was extracted with ether $(50 \mathrm{~mL} \times 3)$. The organic layer was dried $\left(\mathrm{MgSO}_{4}\right)$ and evaporated and the residue was subjected to flash chromatography to get $1.36 \mathrm{~g}$ (71 \%) of a colorless oil. 11: IR (neat): 3300, 2967, 2918, 2854, 2115, 1668, 1442, 1377, 1356, 1264, $1248 \mathrm{~cm}^{-1}$; ${ }^{1} \mathrm{H}$ NMR (500 MHz, $\left.\mathrm{CDCl}_{3}\right)$ : 1.60 (s, 3H), 1.68 (s, 3H), 1.69 (s, 3H), 2.02-2.08 (m, 2H), 2.09-2.15 (m, 2H), 2.41 (t, J = 2.4 Hz, H), 4.09 (d, J = 6.9 Hz, 2H), $4.12(\mathrm{~d}, \mathrm{~J}=2.4 \mathrm{~Hz}, 2 \mathrm{H}), 5.09(\mathrm{t} \mathrm{br}, \mathrm{J}=6.9 \mathrm{~Hz}, \mathrm{H}), 5.33$ ( $\mathrm{tbr}, \mathrm{J}=6.9 \mathrm{~Hz}, \mathrm{H}) ;{ }^{13} \mathrm{C}\left(\mathrm{CDCl}_{3}\right)$ : $\square 16.9,18.1$, $26.1,26.8,40.0,57.1,66.4,74.5,80.5,120.3,124.3,132.1,142.0 ; \mathrm{GC}\left(70{ }^{\circ} \mathrm{C} / 5 \mathrm{~min}-20^{\circ} \mathrm{C} / \mathrm{min}-250\right.$ $\left.{ }^{\circ} \mathrm{C}\right) ; \mathrm{t}_{\mathrm{r}}=11.3 \min (98 \%)$.

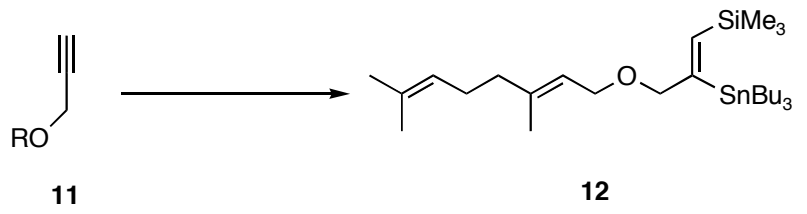

Silylstannylation of 11. To a solution of $\mathrm{Pd}_{2}(\mathrm{dba})_{3}$ $(0.9 \mathrm{mg}, 0.0010 \mathrm{mmol} \mathrm{Pd})$ and $\mathrm{PCy}_{3}(0.0020 \mathrm{mmol})$ in $1 \mathrm{~mL}$ of $\mathrm{C}_{6} \mathrm{D}_{6}$ was added $\mathrm{Bu}_{3} \mathrm{Sn}_{-}-\mathrm{SiMe}_{3}(40 \mathrm{mg}, 0.11$ mmol) followed by the eneyne $11(19.2 \mathrm{mg}, 0.100 \mathrm{mmol})$. The reaction was monitored by ${ }^{1} \mathrm{H}$ NMR. Among the various ligands used $\left(\mathrm{PCy}_{3}, \mathrm{P}\left(\mathrm{C}_{6} \mathrm{~F}_{5}\right)_{3}, \mathrm{PPh}_{3}, \mathrm{P}(\mathrm{OPh})_{3}\right.$, and without ligand), the use of $\mathrm{PCy}_{3}$ gave the highest conversion. The solvent was evaporated from the reaction mixture and the residue was subjected to column chromatography (Hex, $100 \%$ ) to get a colorless oil (48.7 mg, $90 \%$ ). 12: IR (neat): 2956, 2871, 1726, 1591, 1463, 1377, 1247, 1156, 1080, $844 \mathrm{~cm}^{-1} ;{ }^{1} \mathrm{H}$ NMR (500 MHz, $\left.\mathrm{CDCl}_{3}\right)$ : $\square 0.12(\mathrm{~s}, 9 \mathrm{H}), 0.82-1.00(\mathrm{~m}, 15 \mathrm{H}), 1.32-1.40(\mathrm{~m}, 6 \mathrm{H}), 1.40-1.56(\mathrm{~m}, 6 \mathrm{H}), 1.61(\mathrm{~s}, 3 \mathrm{H}), 1.65(\mathrm{~s}, 3 \mathrm{H})$, $1.68(\mathrm{~s}, 3 \mathrm{H}), 2.00-2.06(\mathrm{~m}, 4 \mathrm{H}), 3.95(\mathrm{~d}, \mathrm{~J}=6.7 \mathrm{~Hz}, 2 \mathrm{H}), 4.04\left(\mathrm{~s}, J_{\mathrm{Sn}-\mathrm{H}}=36 \mathrm{~Hz}, 2 \mathrm{H}\right), 5.11(\mathrm{t} \mathrm{br}, \mathrm{J}=6.7$ $\mathrm{Hz}, \mathrm{H}), 5.35(\mathrm{t} \mathrm{br}, \mathrm{J}=6.2 \mathrm{~Hz}, \mathrm{H}), 6.61\left(\mathrm{~s}, J_{\mathrm{Sn}-\mathrm{H}}=180 \mathrm{~Hz}, \mathrm{H}\right) ;{ }^{13} \mathrm{C} \mathrm{NMR}\left(\mathrm{CDCl}_{3}\right)$ : $\square 0.5,11.5,14.1$, 16.9, 18.1, 26.1, 26.9, 27.9, 29.6, 40.0, 66.9, 82.4, 121.6, 124.5, 132.0, 139.6, 144.0, 162.6; HRMS calcd for $[\mathrm{M}+\mathrm{Na}]^{+}$579.3015, found 579.3017; nOe difference spectra confirmed the double bond geometry shown above.

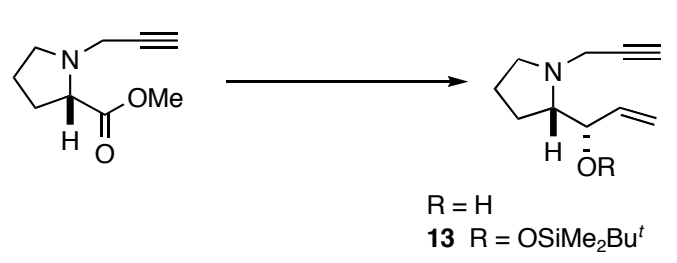

Synthesis of 13 from $N$-propargylpyrrolidine-2carbaldehyde, prepared in situ from $N$-propargyl $L$ proline methyl ester. To a solution of the $N$-propargyl proline methyl ester ${ }^{2}(418 \mathrm{mg}, 2.50 \mathrm{mmol})$ in $5 \mathrm{~mL}$ of toluene

(2) N-Propargyl proline methyl ester: Trost, B. M.; Chen, S.-F. J. Am. Chem. Soc. 1986, 108, 6053. 
was added slowly a solution of DIBAL in toluene $(2.00 \mathrm{~mL}$ of $1.5 \mathrm{M}$ in hexane $3.00 \mathrm{mmol})$ over $1 \mathrm{~h}$, keeping the internal temperature below $-65{ }^{\circ} \mathrm{C}$. After the addition was complete, the mixture was further stirred for $1 \mathrm{~h}$. The reaction was quenched with $1 \mathrm{~mL}$ of $\mathrm{MeOH}$, which was added slowly at <$65{ }^{\circ} \mathrm{C}$. The resulting suspension was poured into an ice-water $(20 \mathrm{~mL})$ with stirring over $15 \mathrm{~min}$. The mixture was made basic to $\mathrm{pH} 13$ using $2 \mathrm{~N} \mathrm{NaOH}$ solution. The aqueous layer was extracted with EtOAc $(20 \mathrm{~mL} \times 3)$ and the organic layer was dried $\left(\mathrm{MgSO}_{4}\right)$ and evaporated, and the residue was dissolved in THF $(10 \mathrm{~mL})$ at $-78^{\circ} \mathrm{C}$. To this solution was added vinyl magnesium bromide $(5.50 \mathrm{~mL}$ of $1.0 \mathrm{M}$ solution in THF) and the reaction mixture was stirred at $-78 \sim 0{ }^{\circ} \mathrm{C}$ for $3 \mathrm{~h}$. The reaction was quenched with saturated $\mathrm{NH}_{4} \mathrm{Cl}$ solution $(10 \mathrm{~mL})$ then made basic to $\mathrm{pH} 8-9$ using $2 \mathrm{~N} \mathrm{NaOH}$ solution. The layers were separated and the aqueous layer was extracted with $\mathrm{Et}_{2} \mathrm{O}(10 \mathrm{~mL} \times 4)$. The combined organic layer was dried $\left(\mathrm{MgSO}_{4}\right)$, evaporated, and the residue was subjected to column chromatography $(\mathrm{EtOAc} / \mathrm{Hex}=1 / 1)$ to get $29.8 \mathrm{mg}(7 \%)$ of the minor isomer and $144 \mathrm{mg}(35 \%)$ of the major isomer along with $159 \mathrm{mg}$ (45\%) of a mixture of two. major isomer: IR (neat): 3418, 3280, 2960, 2890, 2340, 2096, 1706, 1641, 1434, 1330, 1200, 1120, 996, $922 \mathrm{~cm}^{-1}$; ${ }^{1} \mathrm{H}$ NMR (400 MHz, $\left.\mathrm{CDCl}_{3}\right)$ : $\square 1.65-1.88\left(\mathrm{~m}, 2 \mathrm{H}, 3 \mathrm{H}\right.$, ring $\left.\mathrm{CH}_{2}\right), 1.88-1.97\left(\mathrm{~m}, \mathrm{H}\right.$, ring $\left.\mathrm{CH}_{2}\right), 2.21$ (s br, $\left.\mathrm{H}, \mathrm{C} \equiv \mathrm{CH}\right), 2.81$ (m, H, $\left.\mathrm{NCH}_{2} \mathrm{CH}_{2}\right), 2.97$ (m, H, NCH), 3.08 (m, H, $\left.\mathrm{NCH}_{2} \mathrm{CH}_{2}\right), 3.50-3.68\left(\mathrm{~m}, 2 \mathrm{H}, \mathrm{NCH} \mathrm{C}_{2} \mathrm{C}\right), 3.91$ (s br, $\mathrm{CHOH}), 5.16\left(\mathrm{td}, \mathrm{J}=1.6,10.5 \mathrm{~Hz}, \mathrm{H}, \mathrm{CH}=\mathrm{CH}_{2}\right), 5.35\left(\mathrm{td}, \mathrm{J}=1.6,17.2 \mathrm{~Hz}, \mathrm{H}, \mathrm{CH}=\mathrm{CH}_{2}\right), 5.88$ (ddd, J = 5.2, 10.5, 17.2 Hz, H, CH= $\left.\mathrm{CH}_{2}\right) ;{ }^{13} \mathrm{C}$ NMR (125MHz, $\left.\mathrm{CDCl}_{3}\right)$ : $\square 24.8\left(\operatorname{ring} \mathrm{CH}_{2}\right), 29.7$ (ring $\left.\mathrm{CH}_{2}\right), 44.0\left(\mathrm{NCH}_{2} \mathrm{C} \equiv \mathrm{C}\right), 53.8\left(\mathrm{NCH}_{2} \mathrm{CH}_{2}\right), 65.5(\mathrm{NCH}), 72.7(\mathrm{C} \equiv C \mathrm{H}), 75.0(C \mathrm{HOH}), 80.2(C \equiv \mathrm{CH})$, 115.6 $\left(\mathrm{CH}=\mathrm{CH}_{2}\right), 140.2\left(\mathrm{CH}=\mathrm{CH}_{2}\right)$; HRMS calcd for $[\mathrm{M}+\mathrm{H}]^{+} 166.1226$, found 166.1229 .

To a solution of the major allyl alcohol prepared above $(50.0 \mathrm{mg}, 0.304 \mathrm{mmol})$ and imidazole (31 mg, $0.46 \mathrm{mmol})$ in $1 \mathrm{~mL}$ of $\mathrm{CH}_{2} \mathrm{Cl}_{2}$ was added TBDMS-Cl (55 mg, $0.36 \mathrm{mmol}$ ) at rt. The reaction mixture was stirred at $\mathrm{rt}$ for $7 \mathrm{~h}$. The reaction mixture was diluted with $\mathrm{Et}_{2} \mathrm{O}(10 \mathrm{~mL})$ and brine $(10$ $\mathrm{mL})$ was added to the mixture. The aqueous layer was extracted with $\mathrm{Et}_{2} \mathrm{O}(10 \mathrm{~mL} \times 3)$. The organic layer was dried $\left(\mathrm{MgSO}_{4}\right)$, evaporated and the residue was subjected to flash chromatography $(\mathrm{EtOAc} / \mathrm{Hex}=1 / 16)$ to get $66.6 \mathrm{mg}(78 \%)$ of a colorless oil. 13: ${ }^{1} \mathrm{H}$ NMR $\left(500 \mathrm{MHz}, \mathrm{CDCl}_{3}\right): \square 0.05$ (s, 3H, Me), $0.08(\mathrm{~s}, 3 \mathrm{H}, M e), 0.90(\mathrm{~s}, 9 \mathrm{H}, \mathrm{Me}), 1.50-1.78\left(\mathrm{~m}, 4 \mathrm{H}\right.$, ring $\left.\mathrm{CH}_{2}\right), 2.17\left(\mathrm{~s}\right.$ br, $\left.\mathrm{H}, \mathrm{C}_{-} \mathrm{CH}\right)$, $2.60(\mathrm{~m}, \mathrm{H}), 2.78(\mathrm{~m}, \mathrm{H}), 2.98(\mathrm{~m}, \mathrm{H}), 3.56$ (m, 2H), $4.16(\mathrm{~m}, \mathrm{H}), 5.13$ (m, H, J = 10.5 Hz, H), 5.24 (d, $\mathrm{J}=17.2 \mathrm{~Hz}, \mathrm{H}), 5.90$ (ddd, $\mathrm{J}=5.3,10.5 \mathrm{~Hz}, 16.4 \mathrm{~Hz}$ ). 


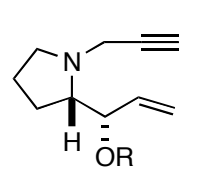

13

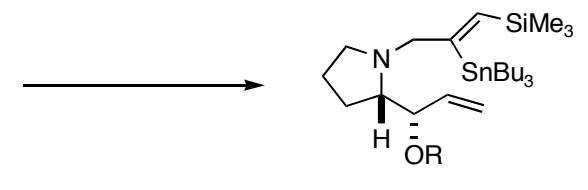

$14 \mathrm{R}=\mathrm{TBS}(41 \%)$

Silylstannylation of 13. To a solution of $\mathrm{Pd}_{2}(\mathrm{dba})_{3}$ (1.9 $\mathrm{mg}, \quad 0.0040 \mathrm{mmol} \mathrm{Pd})$ and tris-(otolyl)phosphine $(2.5 \mathrm{mg}, 0.0080 \mathrm{mmol})$ in $1 \mathrm{~mL}$ of $\mathrm{C}_{6} \mathrm{D}_{6}$ was added $\mathrm{Bu}_{3} \mathrm{Sn}-\mathrm{SiMe}_{3}(67 \mathrm{mg}, 0.18 \mathrm{mmol})$

followed by starting eneyne $13(46.6 \mathrm{mg}, 0.167 \mathrm{mmol})$. The reaction was monitored by ${ }^{1} \mathrm{H}$ NMR. After $24 \mathrm{~h}$ at $60{ }^{\circ} \mathrm{C}$, the ${ }^{1} \mathrm{H}$ NMR spectrum indicated that the starting material had mostly disappeared, and the formation of adduct $\mathbf{1 4}$ was observed. After evaporation of solvent, the residue was subjected to flash chromatography (hex, 100\%) to get $43.8 \mathrm{mg}(41 \%)$ of 14 as a colorless oil. 14: IR (neat): 2956, 2928, 2856, 1642, 1463, 1404, 1376, 1360, 1342, 1249, 1128, 1077, 1030, 1005, 916, 837, 775 $\mathrm{cm}^{-1} ;{ }^{1} \mathrm{H}$ NMR (500 MHz, $\left.\mathrm{CDCl}_{3}\right) \square 0.04$ (s, 3H, TBS), 0.11 (s, 9H+3H, TBS, TMS), 0.89 (s, 9H, TBS), $\mathrm{SnBu}_{3}$ peaks are omitted, 1.52-1.70 (m, 4H), $2.06\left(\mathrm{~m}, \mathrm{H}, \mathrm{NCH}_{2} \mathrm{CH}_{2}\right), 2.61(\mathrm{~m}, \mathrm{H}, \mathrm{NH}), 2.83(\mathrm{t}, \mathrm{J}$ $\left.=6.9 \mathrm{~Hz}, \mathrm{H}, \mathrm{NCH}_{2} \mathrm{CH}_{2}\right), 3.03\left(\mathrm{~d}, \mathrm{~J}=12.6 \mathrm{~Hz}, \mathrm{H}, \mathrm{NCH}_{2} \mathrm{CSn}\right), 3.71$ (d, J = 12.6 Hz, H, NCH $\mathrm{CSn}, 4.28$ $(\mathrm{s}, \mathrm{H}, \mathrm{CHOH}), 5.12\left(\mathrm{~d}, \mathrm{~J}=10.6 \mathrm{~Hz}, \mathrm{H}, \mathrm{CH}=\mathrm{CH}_{2}\right), 5.29\left(\mathrm{~d}, \mathrm{~J}=17.5 \mathrm{~Hz}, \mathrm{H}, \mathrm{CH}=\mathrm{CH}_{2}\right), 6.06(\mathrm{ddd}, \mathrm{J}=$ 2.3, 10.6, $\left.17.3 \mathrm{~Hz}, \mathrm{H}, \mathrm{CH}=\mathrm{CH}_{2}\right), 6.59\left(\mathrm{~s},{ }^{3} \mathrm{~J}_{\mathrm{Sn}-\mathrm{H}}=164 \mathrm{~Hz}, \mathrm{H}, \mathrm{CHSi}\right) ;{ }^{13} \mathrm{C} \mathrm{NMR}\left(125 \mathrm{MHz}, \mathrm{CDCl}_{3}\right)$ : $\square-$ 4.38 (TBS), -4.33 (TBS), 0.7 (TMS), $11.6\left(\mathrm{SnBu}_{3}\right), 14.1\left(\mathrm{SnBu}_{3}\right), 18.7\left(C \mathrm{Me}_{3}\right), 24.5\left(\operatorname{ring} C \mathrm{H}_{2}\right), 25.6$ $\left(\operatorname{ring} \mathrm{CH}_{2}\right), 26.3\left(\mathrm{C}\left(\mathrm{CH}_{3}\right)_{3}\right), 28.0\left(\mathrm{SnBu}_{3}\right), 29.8\left(\mathrm{SnBu}_{3}\right), 55.3\left(\mathrm{NCH}_{2} \mathrm{CH}_{2}\right), 69.3(\mathrm{NCH}), 73.6$ $\left(\mathrm{NCH}_{2} \mathrm{CSn}\right), 73.7(\mathrm{CHOH}), 114.4\left(\mathrm{CH}=\mathrm{CH}_{2}\right), 138.6\left(\mathrm{CH}=\mathrm{CH}_{2}\right), 144.7(C \mathrm{Sn}), 164.5(C \mathrm{Si})$; HRMS calcd for $[\mathrm{M}+\mathrm{Na}]^{+}$666.3519, found 666.3531; nOe difference spectra confirmed the double bond geometry shown above.

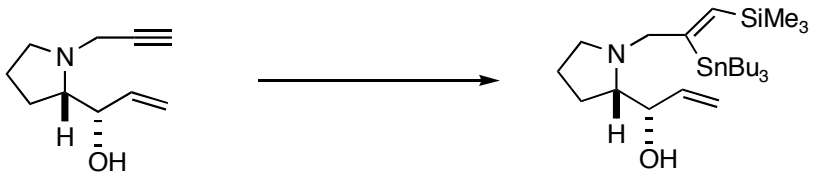

Silylstannylation of a substrate with an unprotected alcohol. To a solution of $\mathrm{Pd}_{2}(\mathrm{dba})_{3}$ (2.9 $\mathrm{mg}, 0.0062 \mathrm{mmol} \mathrm{Pd})$ and $\mathrm{P}\left(\mathrm{C}_{6} \mathrm{~F}_{5}\right)_{3}(6.6 \mathrm{mg}$, $0.012 \mathrm{mmol})$ in $1 \mathrm{~mL}$ of $\mathrm{C}_{6} \mathrm{D}_{6}$ was added $\mathrm{Bu}_{3} \mathrm{Sn}_{-} \mathrm{SiMe}_{3}(100 \mathrm{mg}, 0.275 \mathrm{mmol})$ followed by starting eneyne (41.0 mg, 0.250 mmol). The reaction was monitored by ${ }^{1} \mathrm{H} \mathrm{NMR}$. After 2 days at $60{ }^{\circ} \mathrm{C}$, the ${ }^{1} \mathrm{H}$ NMR spectrum indicated that the conversion into did not go further. The solvent from the mixture was evaporated and the residue was subjected to flash chromatography to get $27.9 \mathrm{mg}(21 \%)$ of the expected product and $30.0 \mathrm{mg}$ (79\%) of starting eneyne. silylstannyl olefin: IR (neat): 3426, 2957, 2872, 1713, 1638, 1463, 1376, 1247, 1123, 1072, 993, 919, 837, $734 \mathrm{~cm}^{-1} ;{ }^{1} \mathrm{H}$ NMR $(500 \mathrm{MHz}$, $\left.\mathrm{CDCl}_{3}\right) \square 0.11$ (s, 9H, TMS), $\mathrm{SnBu}_{3}$ peaks are omitted, $1.61\left(\mathrm{~m}, \mathrm{H}\right.$, ring $\left.\mathrm{CH}_{2}\right), 1.72\left(\mathrm{~m}, 2 \mathrm{H}\right.$, ring $\left.\mathrm{CH}_{2}\right)$, 1.86 (m, H, ring $\mathrm{CH}_{2}$ ), 2.37 (td, J = 7.1, $10.7 \mathrm{~Hz}, \mathrm{H}, \mathrm{NCH}_{2} \mathrm{CH}_{2}$ ), 2.71 (m, H, NCH), 2.89 (ddd, J = 5.8, 6.8, 10.8 Hz, H, $\mathrm{NCH}_{2} \mathrm{CH}_{2}$ ), 3.13 (dd, J = 1.0, 14.0 Hz, J $\mathrm{Sn-H}=40 \mathrm{~Hz}, \mathrm{H}, \mathrm{NCH}_{2} \mathrm{CSn}$ ), 3.48 (s br, H, 
$\mathrm{OH}), 3.56\left(\mathrm{dd}, \mathrm{J}=1.8,14.2 \mathrm{~Hz}, \mathrm{~J}_{\mathrm{Sn}-\mathrm{H}}=22 \mathrm{~Hz}, \mathrm{H}, \mathrm{NCH} \mathrm{CSSn}_{2}, 3.74(\mathrm{t}, \mathrm{J}=6.1 \mathrm{~Hz}, \mathrm{H}, \mathrm{CHOH}), 5.12\right.$ (td, $\left.\mathrm{J}=1.3,10.6 \mathrm{~Hz}, \mathrm{H}, \mathrm{CH}=\mathrm{CH}_{2}\right), 5.29\left(\mathrm{td}, \mathrm{J}=1.4,17.3 \mathrm{~Hz}, \mathrm{H}, \mathrm{CH}=\mathrm{CH}_{2}\right), 5.82(\mathrm{ddd}, \mathrm{J}=5.8,10.5,16.8$ $\left.\mathrm{Hz}, \mathrm{H}, \mathrm{CH}=\mathrm{CH}_{2}\right), 6.62\left(\mathrm{~s},{ }^{3} J_{\mathrm{Sn}-\mathrm{H}}=167 \mathrm{~Hz}, \mathrm{H}, \mathrm{CHSi}\right) ;{ }^{13} \mathrm{C} \mathrm{NMR}\left(125 \mathrm{MHz}, \mathrm{CDCl}_{3}\right): \square 0.6\left(\mathrm{SiMe}_{3}\right)$, $11.5\left(\mathrm{SnBu}_{3}\right), 14.1\left(\mathrm{SnBu}_{3}\right), 24.1\left(\right.$ ring $\left.C \mathrm{H}_{2}\right), 27.6\left(\operatorname{ring} C_{2}\right), 27.9\left(\mathrm{SnBu}_{3}\right), 29.7\left(\mathrm{SnBu}_{3}\right), 53.5$ $\left(\mathrm{NCH}_{2} \mathrm{CH}_{2}\right), 69.8(\mathrm{NCH}), 72.2\left(\mathrm{NCH}_{2} \mathrm{CSn}\right), 74.5(\mathrm{CHOH}), 115.9\left(\mathrm{CH}=\mathrm{CH}_{2}\right), 139.7\left(\mathrm{CH}=\mathrm{CH}_{2}\right), 144.4$ (CSi), 163.1 (CSn); HRMS calcd for $[\mathrm{M}+\mathrm{Na}]^{+}$552.2654, found 552.2643; Selected nOe confirmed the double bond geometry shown.

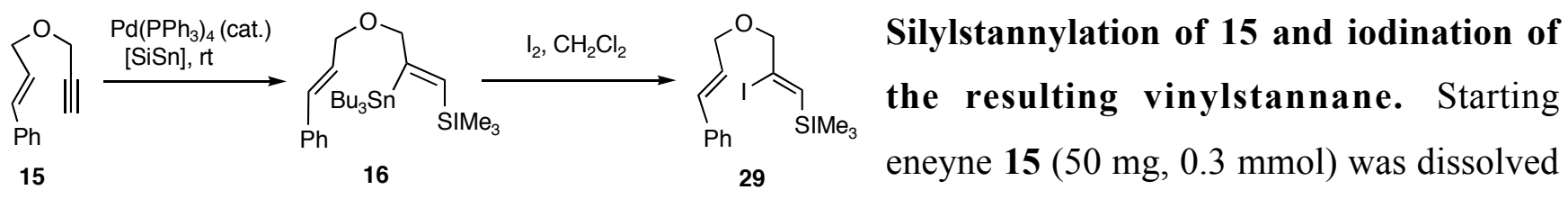
in $1 \mathrm{~mL}$ of benzene in a $2 \mathrm{~mL}$ of micorscale vial. To the solution were added $\mathrm{Pd}\left(\mathrm{PPh}_{3}\right)_{4}(17.0 \mathrm{mg}, 0.01$ mmol, $0.05 \mathrm{eq})$ and $\mathrm{Bu}_{3} \mathrm{SnSiMe}_{3}(0.11 \mathrm{~g}, 0.3 \mathrm{mmol})$. The reaction mixture was allowed to stir for 1 day. The solution was then directly purified by flash column chromatography (hexane/ether $=1 / 1)$ to yield $0.11 \mathrm{~g}$ of silylstannane $(73 \%)$. The cooled $\left(0{ }^{\circ} \mathrm{C}\right)$ solution of silylstannane thus obtained $(0.11 \mathrm{~g}$, $0.2 \mathrm{mmol}$ ) in $\mathrm{CH}_{2} \mathrm{Cl}_{2}$ was titrated with $\mathrm{I}_{2}$ solution in $\mathrm{CH}_{2} \mathrm{Cl}_{2}$. Upon completion of the titration, the solution was concentrated and purified by flash chromatography (hexane/ether $=9 / 1$ ) to yield $73.0 \mathrm{mg}$ of the corresponding iodide (93\%). 29: ${ }^{1} \mathrm{H}$ NMR (400 MHz, $\left.\mathrm{CDCl}_{3}\right) \square 0.16$ (s, 9H, TMS), 4.10 (d, J = $\left.1.5 \mathrm{~Hz}, 2 \mathrm{H}, \mathrm{OCH}_{2} \mathrm{CI}=\mathrm{CHTMS}\right), 4.12\left(\mathrm{~d}, \mathrm{~J}=8.0 \mathrm{~Hz}, 2 \mathrm{H}, \mathrm{OCH}_{2} \mathrm{CH}=\mathrm{CHPh}\right), 6.26(\mathrm{ddd}, \mathrm{J}=6.1,15.8$ $\left.\mathrm{Hz}, \mathrm{H}, \mathrm{OCH}_{2} \mathrm{CH}=\mathrm{CHPh}\right), 6.65\left(\mathrm{~d}, \mathrm{~J}=15.9 \mathrm{~Hz}, \mathrm{H}, \mathrm{OCH}_{2} \mathrm{CH}=\mathrm{CHPh}\right), 6.77\left(\mathrm{~s}, \mathrm{H}, \mathrm{OCH}_{2} \mathrm{CI}=\mathrm{CHTMS}\right)$, 7.19-7.37 (m, 5H); ${ }^{13} \mathrm{C}$ NMR (100 MHz, $\left.\mathrm{CDCl}_{3}\right)$ : $\square 0.0$ (q), 71.9 (t), 81.6 (t), 118.4 (s), 126.5 (d), 127.7 (d), 128.9 (d), 129.7 (d), 134.1 (d), 137.5 (d), 137.7 (s).

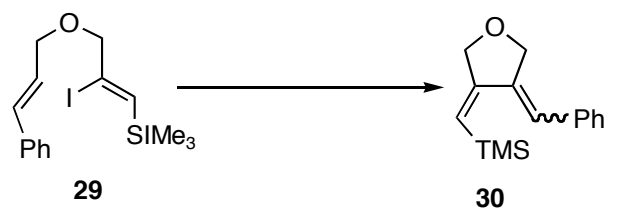

Heck cyclization of 29. Starting iodide (29, $0.15 \mathrm{~g}, 0.4 \mathrm{mmol})$ was dissolved in $1 \mathrm{~mL}$ of $\mathrm{CH}_{3} \mathrm{CN}$ in a $2 \mathrm{~mL}$ microsale vial, fitted with a reflux condensor. To the iodide solution was added $\mathrm{Pd}\left(\mathrm{PPh}_{3}\right)_{4}(22.0 \mathrm{mg}, 0.02 \mathrm{mmol})$ and $\mathrm{Et}_{3} \mathrm{~N}(55.0 \mathrm{mg}, 0.9 \mathrm{mmol}, 2 \mathrm{eq})$. The reaction mixture was refluxed for $4 \mathrm{~h}$. The reaction was then stopped and the solution was directly purified by flash column chromatography (hexane/Et $2 \mathrm{O}=95 / 5)$ to yield $72.0 \mathrm{mg}$ of furan $\mathbf{3 0}$ as a thick clear oil $(72 \%)$. The ratio of $(Z, E)$ to $(Z, Z)$-isomer was determined to be $9: 1$ by ${ }^{1} \mathrm{H}$ NMR spectroscopy. The following ${ }^{1} \mathrm{H}$ NMR describes both (Z,E) and (Z,Z)-isomers. 30: ${ }^{1} \mathrm{H}$ NMR (500 MHz, $\left.\mathrm{C}_{6} \mathrm{D}_{6}\right) \square 0.1$ (s, 9H, TMS, Z,Z), $0.2(\mathrm{~s}, 9 \mathrm{H}, \mathrm{TMS}, \mathrm{Z}, \mathrm{E}), 4.36\left(\mathrm{~s}, 2 \mathrm{H}, \mathrm{OCH}_{2} \mathrm{CR}=\mathrm{CHSiMe}_{3}, \mathrm{Z}, \mathrm{E}\right), 4.51\left(\mathrm{~s}, 2 \mathrm{H}, \mathrm{OCH}_{2} \mathrm{CR}=\mathrm{CHSiMe}_{3}, \mathrm{Z}, \mathrm{E}\right)$, 
$4.68\left(\mathrm{~s}, 2 \mathrm{H}, \mathrm{OCH}_{2} \mathrm{CR}=\mathrm{CHPh}, \mathrm{Z}, \mathrm{Z}\right.$ and $\left.\mathrm{Z}, \mathrm{E}\right), 5.35$ (s, H, C=CHSiMe $\left.3, \mathrm{Z}, \mathrm{E}\right), 6.04$ (s, H, C=CHSiMe 3 , $\mathrm{Z}, \mathrm{E}), 6.81$ (s, H, CR=CHPh, Z,Z), 6.97 (s, H, CR=CHPh, Z,E), 7.00-7.06 (m, 5H, Z,Z and Z,E); ${ }^{13} \mathrm{C}$ NMR (125 MHz, $\mathrm{C}_{6} \mathrm{D}_{6}$ ): $\square 0.4$ (q), 72.6 (t), 75.1 (t), 119.2 (d), 125.2 (d), 127.7 (d), 129.3 (d), 129.4 (d), 137.5 (s), 139.4 (s), 156.1 (s); HRMS calcd for [M] 244.1283, found 244.1280.

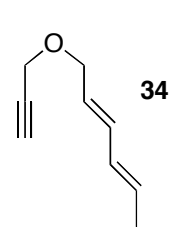

Synthesis of $(2 E, 4 E)$-2,4-hexadienyl 2-propynyl ether (34, entry 7, Table 1). To a precooled $\left(0{ }^{\circ} \mathrm{C}\right)$ suspension of $\mathrm{NaH}(0.54 \mathrm{~g}, 22.3 \mathrm{mmol})$ in THF was added a solution of sorbic alcohol $(2.0 \mathrm{~g}, 20 \mathrm{mmol})$ in $10 \mathrm{~mL}$ of THF from a syringe. The mixture was warmed to room temperature and was stirred for additional $30 \mathrm{~min}$. To the solution was added propargyl bromide $(3.6 \mathrm{~g}, 31.0 \mathrm{mmol})$ via a syringe. The reaction was stopped after $1 \mathrm{~d}$ and was quenched with saturated ammonium chloride. Aqueous layer was extracted with ether (50 mL X 2) and the resulting organic layers were combined, dried and concentrated. The crude product was purified by column chromatography to get $2.1 \mathrm{~g}(75 \%)$ of the pure product identified by comparison of spectral properties with those reported in the literature. ${ }^{3}$

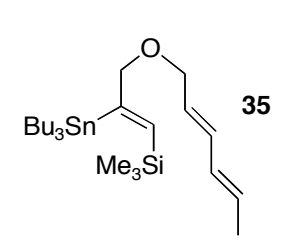

Silylstannylation of (2E, 4E)-2,4-hexadienyl 2-propynyl ether $34\left(35, \mathrm{X}=\mathrm{SnBu}_{3}\right.$, entry 7, Table 1). The propargyl ether $(0.30 \mathrm{~g}, 2.2 \mathrm{mmol})$ was dissolved in $1 \mathrm{~mL}$ of benzene and was placed in a microscale vial. To the solution was added $\mathrm{Pd}\left(\mathrm{PPh}_{3}\right)_{4}$ ( $0.13 \mathrm{~g}, 0.01 \mathrm{mmol}, 0.05$ equiv.) and $\mathrm{Me}_{3} \mathrm{SiSnBu}_{3}$ (0.88 g, $2.4 \mathrm{mmol}, 1.1$ equiv.). The reaction was stopped after 1 day and the solution was concentrated and the product subjected to column chromatography (hexane/ether 95:5). The product $(0.71 \mathrm{~g}, 65 \%)$ was isolated as a thick oil. 35: ${ }^{1} \mathrm{H}$ NMR $\mathrm{Q}\left(\mathrm{CDCl}_{3}\right): 0.12$ (s, 9H, $\left.\mathrm{Si}\left(\mathrm{CH}_{3}\right)_{3}\right), 0.86-1.56$ (m, 27H, Sn-alkyl H), $1.76(\mathrm{~d}, \mathrm{~J}=6.7 \mathrm{~Hz}$, $\left.3 \mathrm{H}, \mathrm{RC}=\mathrm{CHCH}_{3}\right), 3.95\left(\mathrm{~d}, \mathrm{~J}=6.2 \mathrm{~Hz}, 2 \mathrm{H},-\mathrm{O}-\mathrm{CH}_{2} \mathrm{CH}=\mathrm{CHR}\right), 4.04\left(\mathrm{~s}, 2 \mathrm{H}, \mathrm{OCH}_{2} \mathrm{CRSnR}_{3}\right), 5.60-5.73$ $(\mathrm{m}, 2 \mathrm{H},-\mathrm{CH}=\mathrm{CH}-), 6.01-6.24(\mathrm{~m}, 2 \mathrm{H},-\mathrm{CH}=\mathrm{CH}-), 6.61\left(\mathrm{~s}, 1 \mathrm{H},-\mathrm{CSnR}_{3} \mathrm{CHSiMe}_{3}\right) .{ }^{13} \mathrm{C} \mathrm{NMR}^{-}$ $\left(\mathrm{CDCl}_{3}\right): 0.33(\mathrm{q}), 11.3(\mathrm{t}), 13.9(\mathrm{q}), 18.3(\mathrm{q}), 27.7(\mathrm{t}), 29.4(\mathrm{t}), 70.6(\mathrm{t}), 82.3(\mathrm{t}), 127.1(\mathrm{~d}), 129.8(\mathrm{~d} 0$, 131.2 (d0, 133.2 (d), 144.1 (d), 162.0 (s); Anal. Calcd. for $\mathrm{C}_{24} \mathrm{H}_{48} \mathrm{OSnSi}$ C 57.57; H 9.67. Found C $57.85 ; \mathrm{H} 9.69$.

Iodination of $35\left(X=\mathrm{SnBu}_{3}\right.$, entry 7 , Table 1$)$. A cooled solution of $0.12 \mathrm{~g}(0.24 \mathrm{mmol})$ 今 of 35 in $\mathrm{CH}_{2} \mathrm{Cl}_{2}$ was titratred with iodine. Upon completion the reaction mixture was concentrated, and the crude product was purified by flash chromatography on silica gel

(3) Nilsson, Y. I. M.; Gatti, R. G. P.; Andersson, P. G.: Bäckvall, J.-E. Tetrahedron, 1996, 52, 7511. 
using hexane/ether (9:1) as a solvent to get $0.076 \mathrm{~g}(93 \%)$ of the iodide as a clear oil. ${ }^{1} \mathrm{H}$ NMR $\left(\mathrm{C}_{6} \mathrm{D}_{6}\right): 0.15\left(\mathrm{~s}, 9 \mathrm{H}, \mathrm{Si}\left(\mathrm{CH}_{3}\right)_{3}, 1.53\left(\mathrm{~d}, \mathrm{~J}=6.4 \mathrm{~Hz}, 3 \mathrm{H}, \mathrm{RC}=\mathrm{CHCH}_{3}\right), 3.81(\mathrm{~d}, \mathrm{~J}=6.0 \mathrm{~Hz}, 2 \mathrm{H}\right.$, $\left.\mathrm{OCH}_{2} \mathrm{CH}=\mathrm{CHR}\right), 3.97\left(\mathrm{~s}, 2 \mathrm{H}, \mathrm{OCH}_{2} \mathrm{CI}=\mathrm{CHSi}\left(\mathrm{CH}_{3}\right)_{3}\right), 5.41-5.59(\mathrm{~m}, 2 \mathrm{H},-\mathrm{CH}=\mathrm{CH}-)$, 5.89-6.20 (m, $2 \mathrm{H},-\mathrm{CH}=\mathrm{CH}-), 6.92\left(\mathrm{~s}, 1 \mathrm{H},=\mathrm{CHSi}\left(\mathrm{CH}_{3}\right)_{3}\right) ;{ }^{13} \mathrm{C} \mathrm{NMR} \square\left(\mathrm{C}_{6} \mathrm{D}_{6}\right):-0.6,18.4,70.9,80.7,118.9,127.2$, 130.2, 131.8, 133.8, 136.8; HRMS 337.0509 $\left(\mathrm{M}^{=}\right.$calcd for $\left.\mathrm{C}_{12} \mathrm{H}_{21} \mathrm{OISi} 337.0484\right)$.
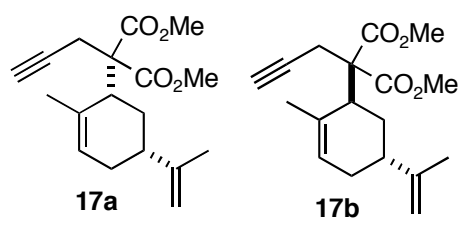

\section{Synthesis of 17a and 17b. Cis-[2-methyl-5-(1-methylethenyl)-2- cyclohexen-1-yl]-2-propynyl-propanedioic acid, dimethyl ester (17a) and Trans-[2-methyl-5-(1-methylethenyl)-2-cyclohexen-1-yl]-2- propanyl-propanedioic acid, dimethyl ester (17b). Di- $O$-methyl}

propargylmalonate $(1.35 \mathrm{~g}, 7.9 \mathrm{mmol})$ was dissolved in minimal amount of THF $(2 \mathrm{~mL}$ and added dropwise to a slurry of $\mathrm{NaH}\left(0.21 \mathrm{~g}, 8.7 \mathrm{mmol}, 1.1\right.$ equiv) dissolved in $10 \mathrm{~mL}$ of THF at $-4{ }^{\circ} \mathrm{C}$. After the slow addition was completed the solution was allowed to stir at room temperature to allow for complete conversion to sodium propargyl malonate. Simultaneously, a solution of $( \pm)$-carveol mesylate $(2.0 \mathrm{~g}, 8.7 \mathrm{mmol}, 1.1 \mathrm{eq}), \mathrm{Pd}\left(\mathrm{PPh}_{3}\right)_{4}(0.45 \mathrm{~g}, 0.4 \mathrm{mmol}, 0.45 \mathrm{eq})$ and $\mathrm{PPh}_{3}(0.41 \mathrm{~g}, 1.6 \mathrm{mmol}$, 0.2 eq) was prepared. To this solution was added the sodium propargyl malonate slurry, dropwise at room temperature. Reaction was allowed to stir for one day, after which it was stopped and quenched with saturated $\mathrm{NH}_{4} \mathrm{Cl}$. Following work-up involved extraction with two portions $(100 \mathrm{~mL})$ of diethyl ether. Careful flash column chromatography (hexane/ether 99:1 as solvent) yielded $1.90 \mathrm{~g} \mathrm{(80 \% )} \mathrm{of}$ cis and trans isomers $\mathbf{1 7} \mathbf{a}$ and $\mathbf{1 7 b}$ in 1:1.2 ratio (w/w after isolating and weighing each compound). 17a. ${ }^{1} \mathrm{H}$ NMR $\square\left(\mathrm{CDCl}_{3}\right): 1.35$ (q, $\mathrm{J}=11.6 \mathrm{~Hz}, 1 \mathrm{H}$, ring $\left.\mathrm{H}\right), 1.66\left(\mathrm{~s}, 3 \mathrm{H}, \mathrm{RCH}=\mathrm{CR}-\mathrm{CH}_{3}\right), 1.70(\mathrm{~s}, 3 \mathrm{H}$, $\left.\mathrm{RCH}=\mathrm{CRCH}_{3}\right), 1.82-1.89(\mathrm{~m}, 2 \mathrm{H}$, ring H's), 1.97-2.05 (m, 1H, ring $\mathrm{H}), 2.05(\mathrm{t}, 1 \mathrm{H}, \mathrm{J}=2.7 \mathrm{~Hz}$, propargyl H), 2.06-2.19 (m, 1H, ring H), 2.74-2.92 (ab q, $\square_{\mathrm{a}}=2.76 \mathrm{ppm}, \square_{\mathrm{b}}=2.89 \mathrm{ppm}, \mathrm{J}=16.8 \mathrm{~Hz}, 2 \mathrm{H}$, $\mathrm{RCH}_{2}-\mathrm{C}\left(\mathrm{CO}_{2} \mathrm{CH}_{3}\right)-\mathrm{R}$ ), 3.27 (s broad, $\left.1 \mathrm{H},-\mathrm{C}\left(\mathrm{CO}_{2} \mathrm{CH}_{3}\right)-\mathrm{CH}-\mathrm{R}_{2}\right), 3.74,3.76$ (two s, $6 \mathrm{H}, \mathrm{CO}_{2} \mathrm{CH}_{3}$ ), 4.67, 4.71 (two s, $\left.2 \mathrm{H}, \mathrm{CH}_{3} \mathrm{Cr}=\mathrm{CH}_{2}\right), 5.56-5.58\left(\mathrm{~m}, 1 \mathrm{H},-\mathrm{CCH}_{3}=\mathrm{CH}-\mathrm{R}\right) ;{ }^{13} \mathrm{C} \mathrm{NMR} \square\left(\left(\mathrm{CDCl}_{3}\right)\right.$ : 20.68, 23.14, 24.11, 30.76, 30.97, 41.62, 44.70, 52.41, 52.53, 60.47, 70.83, 80.13, 108.9, 127.0, 133.3, 149.3, 169.9, 170.9. 17b. ${ }^{1} \mathrm{H}$ NMR $\square\left(\mathrm{CDCl}_{3}\right)$ : 1.56-1.64 (m, $1 \mathrm{H}$, ring $\left.\left.\mathrm{H}\right), 1.69(\mathrm{~s}, 6 \mathrm{H}, \mathrm{RHC}=\mathrm{CRCH})_{3}\right), 1.85-1.90$ (m, 2H, ring H's), 2.01 (t, J = 2.6 Hz, 1H, acetylenic), 2.02-2.04 (m, 1H, ring H), 2.17-2.27 (m, 1H, ring $\left.\mathrm{H}), 2.86\left(\mathrm{~s}, 2 \mathrm{H}, \mathrm{RC}\left(\mathrm{CO}_{2} \mathrm{CH}_{3}\right)-\mathrm{CH}_{2} \mathrm{R}\right), 3.19\left(\mathrm{~s} \text { broad, } 1 \mathrm{H}, \mathrm{C}\left(\mathrm{CO}_{2} \mathrm{CH}_{3}\right)-\mathrm{CHR}\right)_{2}\right), 3.68(\mathrm{~s}, 3 \mathrm{H}$, $\left.\mathrm{CO}_{2} \mathrm{CH}_{3}\right) ; 3.75\left(\mathrm{~s}, 3 \mathrm{H}, \mathrm{CO}_{2} \mathrm{CH}_{3}\right), 4.68,4.70$ (two s, $\left.2 \mathrm{H},-\mathrm{C}=\mathrm{CH}_{2}\right), 5.51\left(\mathrm{~s}, 1 \mathrm{H}, \mathrm{CH}_{3} \mathrm{RC}=\mathrm{CHR}\right) ;{ }^{13} \mathrm{C}$ NMR $\square\left(\mathrm{CDCl}_{3}\right): 21.0,24.64,25.08,30.44,30.77,36.21,41.56,52.61,53.05,60.77,71.46,80.19$, $109.5,126.3,133.7,149.3,171.3,171.5$. 


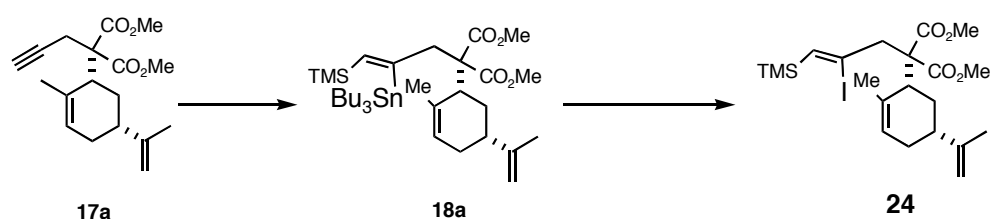

Silylstannylation of $17 \mathrm{a}$ and iodination of 18a. Starting eneyne $17 \mathbf{a}(0.11 \mathrm{~g}, 0.4$ mmol) was dissolved in $1 \mathrm{~mL}$ of benzene and placed in a microscale vial fitted with a reflux condensor. To the solution were added $\mathrm{Bu}_{3} \mathrm{SnSiMe}_{3}$ $(0.20 \mathrm{~g}, 6.0 \mathrm{mmol})$ and $\mathrm{Pd}\left(\mathrm{PPh}_{3}\right)_{4}(23 \mathrm{mg}, 0.02 \mathrm{mmol}, 0.05 \mathrm{eq})$. The solution was heated to $70{ }^{\circ} \mathrm{C}$ for $24 \mathrm{~h}$ after which the reaction was stopped. Crude solution was directly subjected to flash chromatography (hexane/ether $=95 / 5$ ) to yield $0.11 \mathrm{~g}$ of corresponding silylstannane as a thick clear oil $(41 \%)$. Thus obtained silylstannane compound $(0.70 \mathrm{~g}, 1.1 \mathrm{mmol})$ was dissolved in $1 \mathrm{~mL}$ of $\mathrm{CH}_{2} \mathrm{Cl}_{2}$ at $0{ }^{\circ} \mathrm{C}$ and the solution was titrated with iodine in $\mathrm{CH}_{2} \mathrm{Cl}_{2}$ until its color turned from clear to light pink. The solution was concentrated and the crude was subjected to flash chromatography (hexane/ether $=95 / 5)$ to yield $0.53 \mathrm{~g}(94 \%)$ of 24 as a thick clear oil. $24:{ }^{1} \mathrm{H}$ NMR $\left(400 \mathrm{MHz}, \mathrm{CDCl}_{3}\right)$ $\square 0.17$ (s, 9H, TMS), 1.54-1.59 (m, H, ring H), 1.69, 1.72 (s, 6H, RCH=CRMe, $\mathrm{CH}_{2}=\mathrm{CR} M e$ ), 1.85-1.89 (m, 2H, ring $H$ 's), 2.03-2.07 (m, $\mathrm{H}$, ring $H$ ), 2.19 (s br, $\mathrm{H}$, ring $H$ ), 3.16 (s br, $\mathrm{H}, \mathrm{RC}\left(\mathrm{CO}_{2} \mathrm{Me}_{2}-\mathrm{C}_{2} \mathrm{R}_{2}\right.$ ), 3.18-3.59 (Abq, $\square_{\mathrm{a}}=3.2 \mathrm{ppm}, \square_{\mathrm{b}}=3.58 \mathrm{ppm}, \mathrm{J}=15.6 \mathrm{~Hz}, 2 \mathrm{H}, \mathrm{RCH}_{2} \mathrm{CE}_{2} \mathrm{R}$ ), $3.68\left(\mathrm{~s}, 3 \mathrm{H}, \mathrm{CO}_{2} \mathrm{Me}\right.$ ), 3.71 (s, 3H, $\mathrm{CO}_{2} \mathrm{Me}$ ), 4.69 (s, $\left.\mathrm{H}, \mathrm{MeCR}=\mathrm{CH}_{2}\right), 4.73$ (s, H, MeCR=CH ), 5.54 (s, $\left.\mathrm{H}, \mathrm{RC} H=\mathrm{CRMe}\right), 6.43$ (s, $\mathrm{H}, \mathrm{C}=\mathrm{CHTMS}) ;{ }^{13} \mathrm{C}$ NMR (100 MHz, $\left.\mathrm{CDCl}_{3}\right)$ : $\mathrm{C-1.04}$ (q), 21.05 (q), 24.49 (q), 30.21 (t), 30.58 (t), 35.75 (d), 43.83 (d), 52.07 (q), 52.35 (q), 54.31 (t), 62.38 (s), $109.2(\mathrm{t}), 113.4$ (s), 126.5 (d), 133.5 (s), 142.1 (d), 149.2 (s), 170.6 (s), 171.2 (s); Anal. Calcd. for $\mathrm{C}_{21} \mathrm{H}_{33} \mathrm{O}_{4} \mathrm{ISi}$ C, 49.99, H, 6.60 Found: $\mathrm{C}$ $55.60, \mathrm{H}, 6.72$.

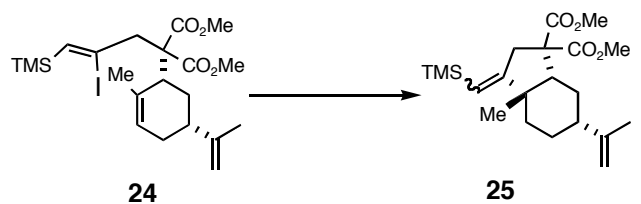

Radical cyclization of 24 . Iodide $24(0.10 \mathrm{~g}, 0.2 \mathrm{mmol})$ was dissolved in $10 \mathrm{~mL}$ of freshly distilled toluene $(0.02 \mathrm{M})$. The solution was placed in $50 \mathrm{~mL}$ three-neck round bottom flask equipped with a reflux condensor. The solution was heated to $100{ }^{\circ} \mathrm{C}$ and the mixture of $n-\mathrm{Bu}_{3} \mathrm{SnH}(86$ $\mathrm{mg}, 0.3 \mathrm{mmol}, 1.5 \mathrm{eq})$ and AIBN (7 mg, $0.04 \mathrm{mmol}), 0.2 \mathrm{eq})$ in toluene $(3 \mathrm{~mL})$ was added to the refluxing solution via syringe pump over $6 \mathrm{~h}$. Conversion appeared to be complete based on TLC (hexane/ether =9:1). After the solvent was removed, the crude was purified by flash chromatography to yield $56 \mathrm{mg}$ of $25(75 \%)$ as a thick colorless oil. The following NMR spectra describe both $\mathrm{Z}$ and $\mathrm{E}$ isomers. (E+Z)-25: ${ }^{1} \mathrm{H}$ NMR (500 MHz, $\left.\mathrm{CDCl}_{3}\right) \square 0.08$ (s, 9H, TMS, E), 0.15 (s, 9H, TMS, Z), 0.80 (q, $\mathrm{H}, \mathrm{J}=12.6 \mathrm{~Hz}$, ring $\mathrm{H}$, E), 0.87 (s, $3 \mathrm{H}, \mathrm{RCH}_{2} \mathrm{CR}_{2} \mathrm{CMe}$, E), 0.98 (s, $3 \mathrm{H}, \mathrm{RCH}_{2} \mathrm{CR}_{2} \mathrm{CMe}, \mathrm{Z}$ ), 1.111.20 (m, $\mathrm{H}$, ring $H, \mathrm{E}$ and $\mathrm{Z}), 1.26-1.31$ (m, $\mathrm{H}$, ring $H, \mathrm{E}$ and $\mathrm{Z}), 1.40-1.57$ (m, 2H, ring $H, \mathrm{E}$ and $\mathrm{Z}$ ), 
$1.64\left(\mathrm{~s}, 3 \mathrm{H}, \mathrm{CH}_{2}=\mathrm{CR} M e, \mathrm{E}\right), 1.66\left(\mathrm{~s}, 3 \mathrm{H}, \mathrm{CH}_{2}=\mathrm{CRMe}, \mathrm{Z}\right), 1.84-1.92$ (m, 2H, ring $H$, E and Z), 2.76 $\left(\mathrm{dd}, \mathrm{J}=2.8,4.9 \mathrm{~Hz}, \mathrm{H}, \mathrm{RC}\left(\mathrm{CO}_{2} \mathrm{Me}\right)_{2} \mathrm{CHR}, \mathrm{E}\right), 3.14-3.45\left(\mathrm{Abq}, \square_{\mathrm{a}}=3.16 \mathrm{ppm}, \square_{\mathrm{b}}=3.43 \mathrm{ppm}, \mathrm{J}=18.5\right.$ $\mathrm{Hz}, 2 \mathrm{H}, \mathrm{RCH}_{2} \mathrm{CE}_{2} \mathrm{R}, \mathrm{E}$ and $\mathrm{Z}$ ), 3.70 , 3.76 (two s, 6H, $\mathrm{CO}_{2} \mathrm{Me}$, E and Z), 4.60, 4.64 (two s, 2H, $\left.\mathrm{MeCR}=\mathrm{CH}_{2}\right), 5.19(\mathrm{~s}, \mathrm{H}, \mathrm{C}=\mathrm{CHSi}, \mathrm{E}), 5.32(\mathrm{~s}, \mathrm{H}, \mathrm{C}=\mathrm{CHSi}, \mathrm{Z}) ;{ }^{13} \mathrm{C} \mathrm{NMR}\left(125 \mathrm{MHz}, \mathrm{CDCl}_{3}\right.$, describes E isomer): -0.12 (q), $20.77(\mathrm{q}), 27.21(\mathrm{t}), 30.75(\mathrm{t}), 31.62(\mathrm{q}), 34.30(\mathrm{t}), 36.52(\mathrm{t}), 44.29(\mathrm{~d}), 48.47(\mathrm{~s})$, 50.46 (d), 52.29 (q), 53.10 (q), 62.35 (s), 108.8 (t), 117.0 (d), 150.2 (s), 161.9 (s), 170.8 (s), 173.1 (s);

${ }^{13} \mathrm{C}$ NMR (125 MHz, $\mathrm{CDCl}_{3}$, describes $\mathrm{Z}$ isomer): $\square$ 0.67, 15.14, 20.44, 26.24, 27.79, 29.86, 32.13, 41.45, 49.4, 49.5, 52.13, 52.52, 68.97, 108.4, 119.2, 144.1, 150.3, 170.6, 172.8; Anal. Calcd. for $\mathrm{C}_{21} \mathrm{H}_{34} \mathrm{O}_{4} \mathrm{Si}$ : C, $66.62 \mathrm{H}, 9.05$, Found: C, $66.62 \mathrm{H} \mathrm{9.05.}$

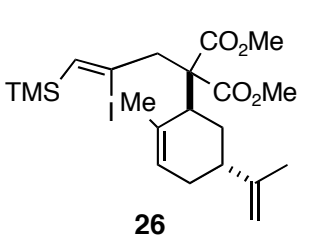

Iodination of 18b. Preparation of 26. Substrate $\mathbf{1 8 b}(0.70 \mathrm{~g}, 1.1 \mathrm{mmol})$ was dissolved in $1 \mathrm{~mL}$ of $\mathrm{CH}_{2} \mathrm{Cl}_{2}$, placed in an ice bath and stirred. The solution was titrated with iodine $/ \mathrm{CH}_{2} \mathrm{Cl}_{2}$ solution until its color turned from clear to light pink. The color change signaled the end of the reaction, which was proved by TLC chromatography (hexane/ether 95:5 as solvent), with a spot to spot conversion. The solution was concentrated and crude was subjected to flash column chromatography (95/5 Hexane/Ether as solvent) to yield $0.53 \mathrm{~g}$ of 26 as thick clear oil in $93 \%$ yield. ${ }^{1} \mathrm{H} \mathrm{NMR} \square\left(\mathrm{CDCl}_{3}\right): 0.17\left(\mathrm{~s}, 9 \mathrm{H}, \mathrm{Si}\left(\mathrm{CH}_{3}\right)_{3}\right), 1.54-$ $1.59(\mathrm{~m}, 1 \mathrm{H}$, ring $\mathrm{H}), 1.72\left(\mathrm{~s}, 6 \mathrm{H}, \mathrm{RHC}=\mathrm{CR}-\mathrm{CH}_{3}\right), 1.85-1.89(\mathrm{~m}, 2 \mathrm{H}$, ring H's $), .2 .03-2.07(\mathrm{~m}, 1 \mathrm{H}$, ring $\mathrm{H}$ ), 2.19 (broad s, $1 \mathrm{H}$, ring $\mathrm{H}), 3.16$ (broad s, $\left.1 \mathrm{H}, \mathrm{RC}\left(\mathrm{CO}_{2} \mathrm{CH}_{3}\right)_{2}-\mathrm{CHR}\right), 3.18-3.59\left(\mathrm{ab} \mathrm{q}, \square_{\mathrm{a}}=3.2\right.$ ppm, $\square_{\mathrm{b}}=3.58$ ppm, J = 15.6 Hz, 2H, $\left.\mathrm{RCH}_{2} \mathrm{C}\left(\mathrm{CO}_{2} \mathrm{CH}_{3}\right)_{2} \mathrm{R}\right), 3.68,3.71\left(\mathrm{~s}, 6 \mathrm{H}, \mathrm{CO}_{2} \mathrm{CH}_{3}\right), 4.69,4.73$ (two s, $\left.2 \mathrm{H}, \mathrm{CH}_{3} \mathrm{CR}=\mathrm{CH}_{2}\right), 5.54\left(\mathrm{~s}, 1 \mathrm{H}, \mathrm{RCH}=\mathrm{CRCH}_{3}\right), 6.43\left(\mathrm{~s}, 1 \mathrm{H}, \mathrm{R}=\mathrm{C} H \mathrm{Si}\left(\mathrm{CH}_{3}\right)_{3}\right){ }^{13} \mathrm{C} \mathrm{NMR} \square$ $\left(\mathrm{CDCl}_{3}\right)$ : $-1.04(\mathrm{q}), 54.31(\mathrm{t}), 62.38(\mathrm{~s}), 109.2(\mathrm{t}), 113.4(\mathrm{~s}), 126.5(\mathrm{~d}), 133.5(\mathrm{~s}), 142.1(\mathrm{~d}), 149.2(\mathrm{~s})$, 170.6 (s), 171.2 (s); Anal. Calcd. for $\mathrm{C}_{21} \mathrm{H}_{33} \mathrm{O}_{4} \mathrm{ISi} \mathrm{C}, 49.99 \mathrm{H}, 6.60$ Found: C, 55.60 H, 6.72.

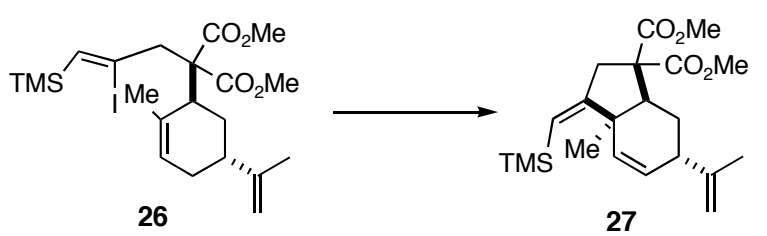

Heck cyclization of 26. Iodide $26(0.10 \mathrm{~g}, 0.2 \mathrm{mmol})$ was dissolved in $1 \mathrm{~mL}$ of $\mathrm{CH}_{3} \mathrm{CN} /$ methyl acrylate $(1: 1)$ mixture and was placed in a $2 \mathrm{~mL}$ of microscale vial fitted with a reflux condensor. To the mixture was added $\mathrm{Pd}\left(\mathrm{PPh}_{3}\right)_{4}(10 \mathrm{mg}, 0.01 \mathrm{mmol}, 0.05 \mathrm{eq})$, and $\mathrm{Et}_{3} \mathrm{~N}(0.04 \mathrm{~g}, 0.4 \mathrm{mmol}, 2 \mathrm{eq})$. The mixture was refluxed overnight, and then the reaction was stopped and the solution was concentrated. Flash chromatography (hexane/ether, 9:1) yielded $61 \mathrm{mg}$ (82\%) of thick clear oil. 27: ${ }^{1} \mathrm{H}$ NMR (400 MHz, $\mathrm{CDCl}_{3}$ ): $\square 0.19$ (s, 9H, TMS), 1.33 (s, 3H, $\left.\mathrm{RCH}=\mathrm{CHCH}_{3} \mathrm{R}\right), 1.56-1.60$ (m, H, ring $\left.H\right), 1.62(\mathrm{~s}, 3 \mathrm{H}$, 
$\mathrm{CH}_{2}=\mathrm{CRMe}$ ), 1.77-1.82 (m, $\mathrm{H}$, ring $\left.H\right), 2.63-2.65$ (m, $\left.\mathrm{H}, \mathrm{CH}_{2}=\mathrm{CCH}_{3} \mathrm{CHR}_{2}\right), 3.16-3.76\left(\mathrm{Abq}, \square_{\mathrm{a}}=3.18\right.$ ppm, $\left.\square_{\mathrm{b}}=3.74 \mathrm{ppm}, \mathrm{J}=16.8 \mathrm{~Hz}, 2 \mathrm{H}, \mathrm{RCH}_{2} \mathrm{C}\left(\mathrm{CO}_{2} \mathrm{Me}\right)_{2} \mathrm{R}\right), 3.24(\mathrm{dd}, \mathrm{J}=9.7,4.4 \mathrm{~Hz}, \mathrm{H}$, $\mathrm{RC}\left(\mathrm{CO}_{2} \mathrm{Me}\right)_{2} \mathrm{CHR}_{2}$ ), 3.29, 3.31 (two s, 6H, $\mathrm{CO}_{2} \mathrm{Me}$ ), 4.85-4.86 (two s, $\mathrm{CH}_{3} \mathrm{CR}=\mathrm{CH}_{2}$ ), 5.46 (dd, J = 10.0, 4.2 Hz, H, R $\left.2 \mathrm{CHCH}=\mathrm{CHCR}_{2} \mathrm{Me}\right), 5.93\left(\mathrm{dd}, \mathrm{J}=10.0,1.8 \mathrm{~Hz}, \mathrm{H}, \mathrm{R}_{2} \mathrm{CHCH}=\mathrm{CHCR}_{2} \mathrm{Me}\right) ;{ }^{13} \mathrm{C}$ NMR (100 MHz, $\left.\mathrm{CDCl}_{3}\right)$ : $\square 0.0,19.8,25.5,27.0,38.4,42.7,46.0,46.4,50.1,50.5,59.4,110.4,119.0$, 126.9, 133.3, 145.1, 163.7, 168.9, 170.7; Anal. Calcd. for $\mathrm{C}_{21} \mathrm{H}_{32} \mathrm{O}_{4} \mathrm{Si}$ C, $66.98 \mathrm{H}, 8.57$ Found: C, $67.10 \mathrm{H}, 8.67$.

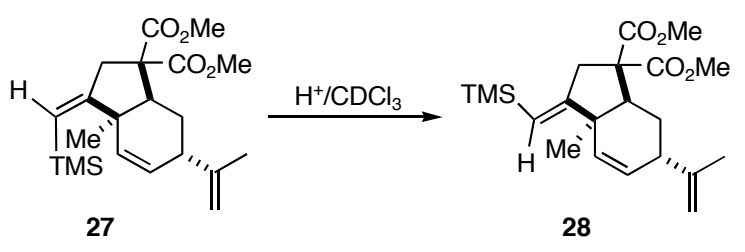

28 wass quantitative after two days. No isolation of this product was performed. 28: ${ }^{1} \mathrm{H}$ NMR (400 $\left.\mathrm{MHz}, \mathrm{CDCl}_{3}\right): \square 0.06(\mathrm{~s}, 9 \mathrm{H}, \mathrm{TMS}), 1.10\left(\mathrm{~s}, 3 \mathrm{H}, \mathrm{RCH}=\mathrm{CHCH}_{3} \mathrm{R}\right), 1.39-1.61\left(\mathrm{~m}, 4 \mathrm{H},-\mathrm{CH}_{2}\right), 1.74(\mathrm{~s}$, $\left.3 \mathrm{H}, \mathrm{CH}_{2}=\mathrm{CR} M e\right), 2.54-2.59\left(\mathrm{~m}, \mathrm{H}, \mathrm{CH}_{2}=\mathrm{CCH}_{3} \mathrm{CHR}_{2}\right), 3.01(\mathrm{dd}, \mathrm{J}=10.3,5.4 \mathrm{~Hz}, \mathrm{H}$, $\mathrm{RCH}_{2} \mathrm{C}\left(\mathrm{CO}_{2} \mathrm{Me}\right)_{2} \mathrm{R}$ ), 3.71, 3.72 (two s, 6H, $\mathrm{CO}_{2} \mathrm{Me}$ ), 4.69, 4.81 (two s, $2 \mathrm{H}, \mathrm{CH}_{3} \mathrm{CR}=\mathrm{CH}_{2}$ ), 5.19 (s, H, $\mathrm{R}=\mathrm{CHSi}), 5.58-5.68\left(\mathrm{~m}, 2 \mathrm{H}, \mathrm{RCH}=\mathrm{CHCH}_{3} \mathrm{R}\right) ;{ }^{13} \mathrm{C}\left(100 \mathrm{MHz}, \mathrm{CDCl}_{3}\right): \square$ 0.0, 16.8, 22.6, 27.6, 28.3, $40.8,46.0,51.5,53.0,68.8,112.8,118.7,128.7,133.5,147.2,152.7,172.4,172.5$.

\section{Epoxide-mediated Cyclizations.}

$\mathrm{Cp}_{2} \mathrm{TiCl}$ was prepared in situ by stirring a $\mathrm{THF}$ solution of $\mathrm{Cp}_{2} \mathrm{TiCl}_{2}$ and excess zinc powder. The supernatant solution was transferred at room temperature to the reaction flask by cannula under nitrogen.

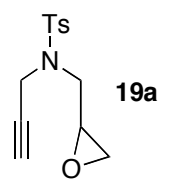

4-Methyl- $N$-oxiranylmethyl- $N$-prop-2-ynyl-benzenesulfonamide(19a). To a solution of 4-methyl- $N$-allyl- $N$-prop-2-ynyl benzenesulfonamide $(0.50 \mathrm{~g}, 2.0 \mathrm{mmol})$ in $\mathrm{CH}_{2} \mathrm{Cl}_{2}(30 \mathrm{~mL})$ MCPBA (0.32 g, $6.9 \mathrm{mmol})$ was added at room temperature. The mixture was stirred for 24 h. The mixture was extracted with $10 \%$ aq. $\mathrm{K}_{2} \mathrm{CO}_{3}(4 \times 10 \mathrm{~mL})$. The combined aqueous phase was extracted with ether $(1 \times 20 \mathrm{~mL})$. The combined organic phase was dried with $\mathrm{MgSO}_{4}$ and concentrated to give a viscous oil which was purified by chromatography on silica gel (hexanes : ether 2:3) to give 19a as a white solid (0.48g, 92\%). mp $76-77^{\circ} \mathrm{C}$; TLC $\mathrm{R}_{\mathrm{f}} 0.20$ (hexanes-ether $1: 1$ ); ${ }^{1} \mathrm{H}$ $\operatorname{NMR}\left(\mathrm{CDCl}_{3}, 500 \mathrm{MHz}\right) \square 7.73(\mathrm{~d}, \mathrm{~J}=8.5 \mathrm{~Hz}, 2 \mathrm{H}), 7.30(\mathrm{~d}, \mathrm{~J}=8.5 \mathrm{~Hz}, 2 \mathrm{H}), 4.24(\mathrm{~d}, \mathrm{~J}=2.5 \mathrm{~Hz}, 2 \mathrm{H})$, 
$3.53(1 / 2 \mathrm{ABqd}, \mathrm{J}=14.5,3.5 \mathrm{~Hz}, 1 \mathrm{H}), 3.25(1 / 2 \mathrm{ABqd}, \mathrm{J}=14.5,6.01 \mathrm{H}), 3.16-3.14(\mathrm{~m}, 1 \mathrm{H}), 2.79(\mathrm{t}, \mathrm{J}$ $=4.5,1 \mathrm{H}), 2.63(\mathrm{dd}, \mathrm{J}=5.0,2.5 \mathrm{~Hz}, 1 \mathrm{H}) ;{ }^{13} \mathrm{C} \mathrm{NMR}\left(\mathrm{CDCl}_{3}, 125 \mathrm{MHz}\right) \square 143.2,137.2,129.5,128.1$, 77.3, 73.8, 50.2, 48.7, 44.4, 37.9, 21.1; IR (KBr, cm $\left.{ }^{-1}\right) 3265,2115,1598,1497,1431,1384,1326$, 1158, 898; MS (ES) calcd for $\mathrm{C}_{13} \mathrm{H}_{15} \mathrm{NO}_{3} \mathrm{SNa}^{+} 288.066482$ found 288.064681 .

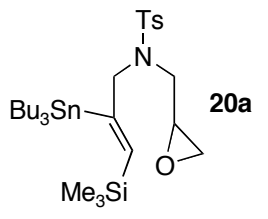

Silylstannylation of 19a. To a solution of 19a $(0.27 \mathrm{~g}, 1.0 \mathrm{mmol})$ in THF $(5 \mathrm{~mL})$ was added $\mathrm{Bu}_{3} \mathrm{SnSiMe}_{3}(0.43 \mathrm{~g}, 1.2 \mathrm{mmol})$ and $\mathrm{Pd}\left(\mathrm{PPh}_{3}\right)_{4}(0.023 \mathrm{~g}, 0.02 \mathrm{mmol})$. The mixture was refluxed for $24 \mathrm{~h}$ under nitrogen atmosphere. The reaction mixture was cooled, concentrated and purified by chromatography on silica gel (hexanes:ether 1:1) to provide 20a as a colorless oil $(0.55 \mathrm{~g}, 88 \%)$. TLC $\mathrm{R}_{\mathrm{f}} 0.50$ (hexanes:ether $\left.3: 1\right) ;{ }^{1} \mathrm{H} \mathrm{NMR}\left(\mathrm{CDCl}_{3}, 500 \mathrm{MHz}\right) \square 7.68$ $(\mathrm{d}, \mathrm{J}=8.5 \mathrm{~Hz}, 2 \mathrm{H}), 7.27(\mathrm{~d}, \mathrm{~J}=8.5 \mathrm{~Hz}, 2 \mathrm{H}), 6.28(\mathrm{t}, \mathrm{J}=1.8 \mathrm{~Hz}, 1 \mathrm{H}), 4.12(\mathrm{td}, \mathrm{J}=8.1,1.2 \mathrm{~Hz}, 2 \mathrm{H})$, $3.24(1 / 2 \mathrm{ABqd}, \mathrm{J}=15.0,4.8 \mathrm{~Hz}, 1 \mathrm{H}), 3.10(1 / 2 \mathrm{ABqd}, \mathrm{J}=15.0,5.5 \mathrm{~Hz}, 1 \mathrm{H}), 3.03-3.00(\mathrm{~m}, 1 \mathrm{H}), 2.69$ $(\mathrm{t}, \mathrm{J}=4.4 \mathrm{~Hz}, 1 \mathrm{H}), 2.44-2.41(\mathrm{~m}, 4 \mathrm{H}), 1.49-1.45(\mathrm{~m}, 6 \mathrm{H}), 1.34-1.30(\mathrm{~m}, 6 \mathrm{H}), 0.99-0.96(\mathrm{~m}, 6 \mathrm{H}), 0.91-$ 0.88 (m, 9H), 0.01 (s, 9H); ${ }^{13} \mathrm{C}$ NMR $\left(\mathrm{CDCl}_{3}, 125 \mathrm{MHz}\right) \square 156.0,143.4,142.6,137.2$, 129.7, 127.4, $60.2,50.3,50.1,46.1,29.2,27.5,21.6,13.78,11.1,0.2 ;{ }^{119} \mathrm{Sn}\left(\mathrm{CDCl}_{3}, 186.4 \mathrm{MHz}\right) \square-54.80$; IR (neat, $\mathrm{cm}^{-1}$ ) 2923, 1598, 1463, 1346, 1246, 1160, 1091, 838; HRMS (ES) calcd for $\mathrm{C}_{8} \mathrm{H}_{51} \mathrm{NO}_{3} \mathrm{SiSnNa}^{+}$: 652.226748; found: 652.226748 .

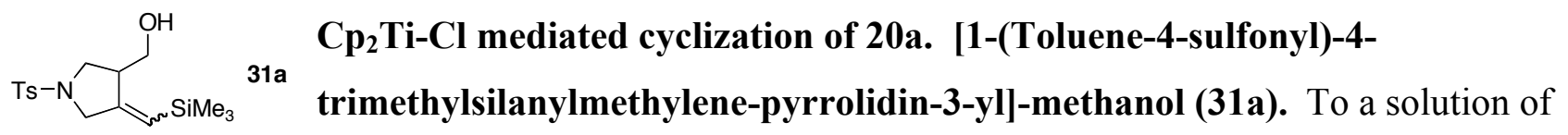
20a $(1.0 \mathrm{~g}, 1.6 \mathrm{mmol})$ was added of $\mathrm{Cp}_{2} \mathrm{TiCl}(1.3 \mathrm{mmol})$ in THF $(40 \mathrm{~mL})$ dropwise over $20 \mathrm{~min}$. The brown mixture was stirred for additional $30 \mathrm{~min}$ and quenched with $0.1 \mathrm{M}$ aqueous $\mathrm{HCl}$. The reaction mixture was concentrated, the residue diluted with ether, organic layer separated and aqueous layer was extracted with ether $(4 \times 10 \mathrm{~mL})$. The combined organic phase was dried with $\mathrm{MgSO}_{4}$ and concentrated to give a reddish oil. This was purified by chromatography on silica gel (hexanes:ether $1: 2)$ to provide 31a ( $\mathrm{E} / \mathrm{Z}$ isomeric mixture) as a thick colorless oil $(0.45 \mathrm{~g}, 78 \%)$. TLC $\mathrm{R}_{\mathrm{f}} 0.23$ (hexanes-ether 1:1); ${ }^{1} \mathrm{H}$ NMR of isomeric mixture $\left(\mathrm{CDCl}_{3}, 500 \mathrm{MHz}\right) \square 7.71(\mathrm{~d}, \mathrm{~J}=7.0,2 \mathrm{H}), 7.33(\mathrm{~d}, \mathrm{~J}$ $=7.0,2 \mathrm{H}), 5.46(\mathrm{~d}, \mathrm{~J}=4.0 \mathrm{~Hz}, 1 \mathrm{H}), 3.97(\mathrm{dt}, \mathrm{J}=14.0,2.0 \mathrm{~Hz}, 1 \mathrm{H}), 3.65(\mathrm{~d}, \mathrm{~J}=10.0 \mathrm{~Hz}, 1 \mathrm{H}), 3.55$ $(\mathrm{dd}, \mathrm{J}=14.0,2.0 \mathrm{~Hz}, 1 \mathrm{H}), 3.50-0.44(\mathrm{~m}, 2 \mathrm{H}), 3.08(\mathrm{dd}, \mathrm{J}=10.0,6.5 \mathrm{~Hz}, 1 \mathrm{H}), 2.87$ (q, $7.0 \mathrm{~Hz}, 1 \mathrm{H})$, 2.43 (s, 3H), 1.79 (s, $1 \mathrm{H}), 0.08$ (s, 9H); ${ }^{13} \mathrm{C}$ NMR $\left(\mathrm{CDCl}_{3}, 125 \mathrm{MHz}\right) \square 151.7,143.6,132.6,129.6$, 127.8, 127.7, 124.4, 63.9, 54.0, 50.6, 45.4, 21.5, 0.0, -0.1. IR (neat, $\mathrm{cm}^{-1}$ ) 3541, 2952, 1636, 1596, 
1338, 1248, 1163, 1092, 1040, 839; HRMS(ES) calcd for $\mathrm{C}_{16} \mathrm{H}_{25} \mathrm{NO}_{3} \mathrm{SSiNa}^{+}: 362.121659$; found 362.122649.

2-Oxiranylmethyl-2-(2-tributylstannanyl-3-trimethylsilanyl-allyl)-malonic acid
diethyl ester (20b). To a solution of $\mathbf{1 9 b}(0.254 \mathrm{~g}, 1.0 \mathrm{mmol})$ in $\mathrm{THF}(5 \mathrm{~mL})$ was
$\mathrm{MeO}_{2} \mathrm{C}$ mixture was refluxed for $24 \mathrm{~h}$ under nitrogen atmosphere. The reaction mixture was cooled, concentrated and purified by chromatography (hexanes-ether 5:1) on silica gel to provide $20 \mathrm{~b}(0.56 \mathrm{~g}$, $90 \%$ ) as a colorless oil. TLC $\mathrm{R}_{\mathrm{f}} 0.38$ (hex: ether 1:1 ); ${ }^{1} \mathrm{H}$ NMR $\left(\mathrm{C}_{6} \mathrm{D}_{6}, 500 \mathrm{MHz}\right) \square 6.68(\mathrm{~s}, 1 \mathrm{H}), 4.11$ $3.98(\mathrm{~m}, 4 \mathrm{H}), 3.38-3.35(\mathrm{~m}, 2 \mathrm{H}), 3.18-3.14(\mathrm{~m}, 1 \mathrm{H}), 2.49(\mathrm{dd}, \mathrm{J}=14.0,4.5 \mathrm{~Hz}, 1 \mathrm{H}), 2.36(\mathrm{dd}, \mathrm{J}=5.0$, $4.0 \mathrm{~Hz}, 1 \mathrm{H}), 2, .21(\mathrm{dd}, \mathrm{J}=14.5,7.0 \mathrm{~Hz}, 1 \mathrm{H}), 2.16(\mathrm{dd}, \mathrm{J}=5.0,2.5 \mathrm{~Hz}, 1 \mathrm{H}), 1.75-1.61(\mathrm{~m}, 6 \mathrm{H}), 1.48-$

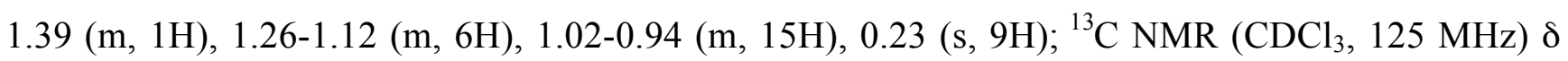
$156.0,143.4,142.6,137.2,129.8,127.4,60.3,50.1,46.1,29.2,27.5,21.6,13.8,11.1,0.2 ;{ }^{119} \mathrm{Sn}$ $\left(\mathrm{CDCl}_{3}, 186.4 \mathrm{MHz}\right)-54.80$; IR (neat, $\left.\mathrm{cm}^{-1}\right)$ 2955, 1731, 1464, 1247, 1097, 838; HRMS(ES) calcd for $\mathrm{C}_{28} \mathrm{H}_{54} \mathrm{O}_{5} \mathrm{SiSnNa}^{+}:$641.265490; found 641.26487.

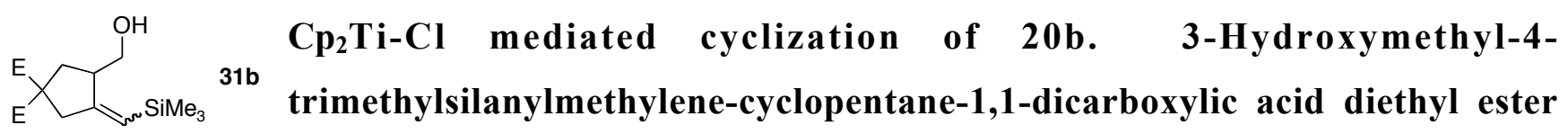
(31b). To a solution of $\mathbf{2 0 b}(0.40 \mathrm{~g}, 0.65 \mathrm{mmol})$ in THF $(2 \mathrm{~mL}), \mathrm{Cp}_{2} \mathrm{TiCl}(0.85 \mathrm{mmol})$ in THF (10 $\mathrm{mL}$ ) was added dropwise. The resulting brown mixture was stirred for $30 \mathrm{~min}$ at room temperature and quenched with $0.1 \mathrm{M}$ aqueous $\mathrm{HCl}$. The reaction mixture was concentrated, the residue diluted with ether, organic layer separated and aqueous layer was extracted with ether ( $4 \times 10 \mathrm{~mL})$. The combined organic phase was dried with $\mathrm{MgSO}_{4}$ and concentrated to give a reddish oil. This was purified by chromatography on silica gel with (hexanes:ether 1:2) to provide two isomers (combined yield 0.17 g, 73\%). 31b: (E) isomer: Rf 0.57 (ether); ${ }^{1} \mathrm{H}$ NMR $\left(\mathrm{CDCl}_{3}, 500 \mathrm{MHz}\right) \square 5.42$ (s, 1H), 4.21 $4.15(\mathrm{~m}, 4 \mathrm{H}), 3.61-3.56(\mathrm{~m}, 1 \mathrm{H}), 3.47-3.41(\mathrm{~m}, 1 \mathrm{H}), 3.10(1 / 2 \mathrm{ABqd}, \mathrm{J}=16.0,2.0 \mathrm{~Hz}, 1 \mathrm{H}), 2.95-2.89$ $(\mathrm{m}, 1 \mathrm{H}), 2.87(1 / 2 \mathrm{ABq}, \mathrm{J}=16.0,1 \mathrm{H}), 2.53(\mathrm{dd}, \mathrm{J}=16.0,8.0 \mathrm{~Hz}, 1 \mathrm{H}), 2.30(\mathrm{dd}, \mathrm{J}=15.0,4.5 \mathrm{~Hz}, 1 \mathrm{H})$, 1.75 (t, J = 5.5 Hz, 1H), 1.29-1.20 (m, 6H), 0.09 (s, 9H); ${ }^{13} \mathrm{C} \mathrm{NMR}\left(\mathrm{CDCl}_{3}, 125 \mathrm{MHz}\right) \square 172.5,171.8$, 157.5, 124.0, 65.5, 61.8, 61.6, 58.4, 45.1, 45.0, 36.5, 14.2, 14.1, 0.2; IR (neat, $\mathrm{cm}^{-1}$ ) 3490, 2955, 1731, 1630, 1446, 1367, 1248, 1188, 838; HRMS (ES) calcd for $\mathrm{C}_{18} \mathrm{H}_{28} \mathrm{O}_{5} \mathrm{SiNa}^{+} 351.159820$ found 351.159760. ( $Z$ ) isomer: $\mathrm{R}_{\mathrm{f}} 0.50$ (ether); ${ }^{1} \mathrm{H} \mathrm{NMR}\left(\mathrm{CDCl}_{3}, 500 \mathrm{MHz}\right) \square 5.42$ (q, J = 2.5 Hz, 1H), 4.20$4.13(\mathrm{~m}, 4 \mathrm{H}), 3.65(\mathrm{t}, \mathrm{J}=6.0 \mathrm{~Hz}, 2 \mathrm{H}), 3.05(1 / 2 \mathrm{ABq}, \mathrm{J}=16.5 \mathrm{~Hz}, 1 \mathrm{H}), 2.88(1 / 2 \mathrm{ABqt}, \mathrm{J}=17.0,2.5$ 
$\mathrm{Hz}, 1 \mathrm{H}), 2.77-2.73(\mathrm{~m}, 1 \mathrm{H}), 2.56(\mathrm{ddd}, \mathrm{J}=13.0,8.5,1.5 \mathrm{~Hz}, 1 \mathrm{H}), 2.07(\mathrm{dd}, \mathrm{J}=13.5,9.0 \mathrm{~Hz}, 1 \mathrm{H}), 1.56$ $(\mathrm{t}, \mathrm{J}=6.5 \mathrm{~Hz}, 1 \mathrm{H}), 1.25-1.22(\mathrm{~m}, 6 \mathrm{H}), 0.10(\mathrm{~s}, 9 \mathrm{H}) ;{ }^{13} \mathrm{C} \mathrm{NMR}\left(\mathrm{CDCl}_{3}, 125 \mathrm{MHz}\right) \square$ 171.8, 171.7, 157.6, 121.9, 64.6, 61.7, 61.7, 59.1, 47.7, 41.0, 35.9, 14.1, -0.3; IR (neat, $\mathrm{cm}^{-1}$ ) 3425, 2954, 1732, $1624,1446,1367,1249,1190,839$; HRMS (ES) calcd for $\mathrm{C}_{18} \mathrm{H}_{28} \mathrm{O}_{5} \mathrm{SiNa}^{+}: 351.159820$; found 351.159419 .

\section{Examples from Table 1, Supporting Information (not included in Table 1 of printed edition)}

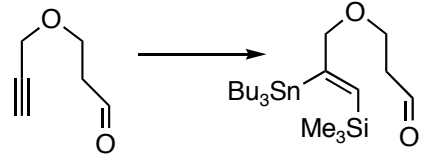

32

Silylstannylation of 32. In a $20 \mathrm{~mL}$ vial, $\mathrm{Pd}_{2}(\mathrm{dba})_{3}(4.6 \mathrm{mg}, 0.010 \mathrm{mmol} \mathrm{Pd})$ and tris-(o-tolyl)phosphine $(6.1 \mathrm{mg}, 0.020 \mathrm{mmol})$ was dissolved in $2 \mathrm{~mL}$ of $\mathrm{C}_{6} \mathrm{D}_{6}$. To the mixture was added $\mathrm{Bu}_{3} \mathrm{Sn}_{-}-\mathrm{SiMe}_{3}(400 \mathrm{mg}, 1.10 \mathrm{mmol})$ followed by alkynyl aldehyde $32(112 \mathrm{mg}, 1.00 \mathrm{mmol})$. The reaction was

monitored by ${ }^{1} \mathrm{H}$ NMR. After $30 \mathrm{~h}$, the reaction was complete, and the solvent was removed and the crude product was subjected to column chromatography $($ EtOAc/Hexane $=1 / 12)$ to get $0.310 \mathrm{~g}(65 \%)$ of $\mathbf{3 3}$ as a pale yellow oil. 33: IR (neat): 2957, 2853, 2726, 1731, 1637, 1561, 1464, 1376, 1247, 1102, $844 \mathrm{~cm}^{-1}$; ${ }^{1} \mathrm{H}$ NMR $\left(500 \mathrm{MHz}, \mathrm{CDCl}_{3}\right)$ : $\square 0.11(\mathrm{~s}, 9 \mathrm{H}), 0.82-0.91(\mathrm{~m}, 15 \mathrm{H}), 1.27-1.40(\mathrm{~m}, 6 \mathrm{H}), 1.40-$ $1.55(\mathrm{~m}, 6 \mathrm{H}), 2.67(\mathrm{td}, \mathrm{J}=6.2,1.9 \mathrm{~Hz}, 2 \mathrm{H}), 3.73(\mathrm{t}, \mathrm{J}=6.2 \mathrm{~Hz}, 2 \mathrm{H}), 4.06(\mathrm{~m}, 2 \mathrm{H}), 6.59\left(\mathrm{~s},{ }^{3} \mathrm{~J}_{\mathrm{Sn}-\mathrm{H}}=174\right.$ $\mathrm{Hz}), 9.80(\mathrm{t}, \mathrm{J}=1.9 \mathrm{~Hz}, \mathrm{H}) ;{ }^{13} \mathrm{C} \mathrm{NMR}\left(125 \mathrm{MHz}, \mathrm{CDCl}_{3}\right)$ : $\square-2.1,8.9,11.5,(\mathrm{Me}), 25.3,27.0,41.8$, $61.6(\mathrm{CCH}), 81.2(\mathrm{CCH}), 142.2,159.0,199.0$; HRMS calcd for $[\mathrm{M}+\mathrm{Na}]^{+}: 499.2025$; found 499.2045. The regiochemistry was confirmed by NOE difference spectra.

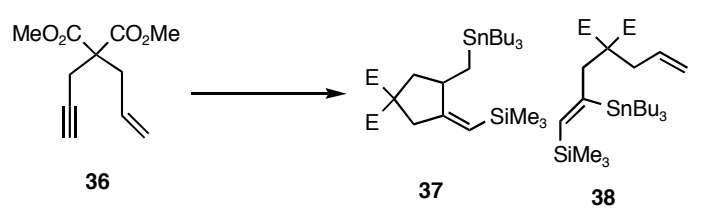

Cyclization of enenynes. Reactions of 36 with silylstannanes. Dependence of simple addition vs. cyclization on reaction conditions. A solution of $101 \mathrm{mg}$ of di-O-methylallylpropargylmalonate $36(0.481 \mathrm{mmol}), 165 \square 1$ of $\mathrm{Me}_{3} \mathrm{SiSnBu}_{3}(0.476 \mathrm{mmol}), 8 \mathrm{mg}$ of $\mathrm{Pd}_{2}(\mathrm{dba})_{3}(0.009 \mathrm{mmol})$ and $25 \mathrm{mg}$ of $\left(\mathrm{C}_{6} \mathrm{~F}_{5}\right)_{3} \mathrm{P}(0.047 \mathrm{mmol})$ in $0.2 \mathrm{~mL}$ of benzene was prepared. The solution was heated at $62{ }^{\circ} \mathrm{C}$ for $20 \mathrm{~h}$. The reaction mixture was then chromatographed on silica gel (hexanes/ether; 95/5. 90/10) to get $136 \mathrm{mg}(49 \%)$ of the cyclized product. 37. ${ }^{1} \mathrm{H}$ NMR (500 MHz, $\left.\mathrm{C}_{6} \mathrm{D}_{6}\right)$ : $\square 0.1$ (s, 9H, TMS), $\mathrm{Bu}_{3} \mathrm{Sn}$ peaks are omitted, $1.76\left(\mathrm{dd}, \mathrm{J}=5.2,13 \mathrm{~Hz}, \mathrm{H}, \mathrm{CH}_{2} \mathrm{C}=\mathrm{CHSiMe}_{3}\right.$ ), $2.70\left(\mathrm{dd}, \mathrm{J}=8.1,19.8 \mathrm{~Hz}, \mathrm{H}, \mathrm{CH}_{2} \mathrm{CHCH}_{2} \mathrm{SnBu}_{3}\right.$ ), 2.73 (d, J = 16.1 Hz, H, $\mathrm{CH}_{2} \mathrm{CHCH}_{2} \mathrm{SnBu}_{3}$ ), 2.79$2.90\left(\mathrm{~m}, \mathrm{H}, \mathrm{CHCH}_{2} \mathrm{SnBu}_{3}\right.$ ), 3.31 (d, J = 16.1 Hz, H, CH $\mathrm{C}_{2} \mathrm{C}=\mathrm{CHSiMe}_{3}$ ), 3.70 (s, 3H, $\mathrm{CO}_{2} \mathrm{Me}$ ), 3.72 (s, $3 \mathrm{H}, \mathrm{CO}_{2} \mathrm{Me}$ ), 5.27 (s, $\mathrm{H}, \mathrm{C}=\mathrm{CHSiMe}$ ) ${ }^{13} \mathrm{C}$ NMR (125 MHz, $\mathrm{CDCl}_{3}$ ): $\square 0.06$ (q), 9.2 (t), 13.6 (q), 19.0 
$(\mathrm{t}), 27.5(\mathrm{t}), 29.1(\mathrm{t}), 40.2(\mathrm{~d}), 42.4(\mathrm{t}), 44.2(\mathrm{t}), 52.5(\mathrm{q}), 58.3(\mathrm{~s}), 119.8(\mathrm{~d}), 164.9(\mathrm{~s}), 172.1(\mathrm{~s}), 172.4$ (s); Anal. calcd for $\mathrm{C}_{26} \mathrm{H}_{50} \mathrm{O}_{4} \mathrm{SiSn} \mathrm{C}, 54.33 \mathrm{H}, 8.78$, Found $\mathrm{C}, 55.10, \mathrm{H}, 8.79$. 38. ${ }^{1} \mathrm{H}$ NMR $\left(\mathrm{CDCl}_{3}\right)$ : $\square$ 0.08 (s, 9H, TMS), 0.84-0.98 (m, 15H, Bu $\left.\mathrm{Sn}_{3} \mathrm{Sn}\right) 1.26-1.53$ (m, 12H, Bu $\left.3 \mathrm{Sn}\right) 2.66$ (d, J = 7.3 Hz, 2H, $\mathrm{CH}_{2} \mathrm{C}=\mathrm{CH}_{2}$ ), 2.89 (s, $2 \mathrm{H}, \mathrm{CH}_{2} \mathrm{CSnBu}_{3}$ ), 3.67 (s, $\left.6 \mathrm{H}, \mathrm{CO}_{2} \mathrm{CH}_{3}\right), 5.03$ (s, broad, $1 \mathrm{H}, \mathrm{CH}_{2} \mathrm{CH}=\mathrm{CH}$ ), 5.07 (s, broad, $\left.1 \mathrm{H}, \mathrm{CH}_{2} \mathrm{CH}=\mathrm{CH}\right), 5.64-5.78\left(\mathrm{~m}, 1 \mathrm{H}, \mathrm{CH}_{2} \mathrm{CH}=\mathrm{CH}\right), 6.32\left(\mathrm{~s}, 1 \mathrm{H},=\mathrm{CHSi}\left(\mathrm{CH}_{3}\right)_{3} ;{ }^{13} \mathrm{C} \mathrm{NMR}\right.$ $\left(\mathrm{CDCl}_{3}\right): \square 0.02(\mathrm{q}), 11.8(\mathrm{t}), 13.5(\mathrm{q}), 19.0(\mathrm{t}), 27.4(\mathrm{t}), 29.0(\mathrm{t}), 37.9(\mathrm{t}), 46.0(\mathrm{t}), 44.2(\mathrm{t}), 51.9(\mathrm{q})$, $59.2(\mathrm{~s}), 111.8(\mathrm{t}), 133.1$ (d), 148.0 (d), $159.2(\mathrm{~s}), 171.3$ (s); HRMS 574.2489, M calcd for $\mathrm{C}_{26} \mathrm{H}_{50} \mathrm{SnSi}$ 574.2500 . 
Table 1. Silylstannylation of Functionalized Acetylenes

\begin{tabular}{|c|c|c|c|c|}
\hline no. & substrate & conditions $^{\mathrm{a}}$ & product & Yield (\%) \\
\hline 1. & 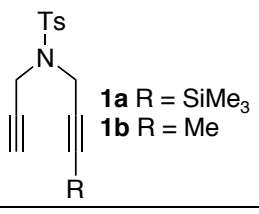 & $\begin{array}{l}\mathrm{Pd}_{2}(\mathrm{dba})_{3} \text { (cat.) } \\
\mathrm{PCy}_{3} \\
\mathrm{C}_{6} \mathrm{H}_{6}, \mathrm{rt}, 16 \mathrm{~h}\end{array}$ & $\mathrm{Bu}_{3} \mathrm{Sn}-\overbrace{\mathrm{Me}_{3} \mathrm{Si}}^{\mathrm{Ts}}$ & $\begin{array}{l}\mathbf{5 a}(88) \\
\mathbf{5 b}(67)\end{array}$ \\
\hline 2. & $\begin{array}{l}\mathrm{Ar} \\
7\end{array}$ & $\begin{array}{l}\mathrm{Me}_{3} \mathrm{SiSnBu}_{3} \\
\mathrm{Pd}_{\left(\mathrm{PPh}_{3}\right)_{4} \text { (cat.) }} \mathrm{C}_{6} \mathrm{H}_{6}, 80^{\circ} \mathrm{C}, 6 \mathrm{~h}\end{array}$ & 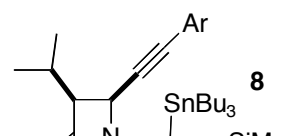 & 54 \\
\hline 3. & $\prod_{\mathrm{CO}_{2} \mathrm{Me}}^{9}$ & $\begin{array}{l}\mathrm{Pd}_{2}(\mathrm{dba})_{3} \\
\mathrm{P}(o-\text { tol })_{3} \\
\mathrm{rt}\end{array}$ & $\int_{\mathrm{Ph}} \mathrm{Bu}_{3} \mathrm{Sn} \overbrace{\mathrm{SIMe}_{3}}^{16}$ & 80 \\
\hline 4. & & $\begin{array}{l}\mathrm{Pd}_{2}(\mathrm{dba})_{3} \text { (cat.) } \\
\mathrm{PCy}_{3}, \mathrm{rt}\end{array}$ & & 90 \\
\hline 5. & 13 & $\begin{array}{l}\mathrm{Pd}_{2}(\mathrm{dba})_{3} \\
\mathrm{P}(o-\text { tol })_{3} \\
60{ }^{\circ} \mathrm{C}, 24 \mathrm{~h}\end{array}$ & $\mathrm{Bu}_{3 \mathrm{Sn}}$ & 41 \\
\hline 6. & $P_{\mathrm{Ph}}$ & $\begin{array}{l}\mathrm{Me}_{3} \mathrm{Si}_{-}-\mathrm{SnBu}_{3} \\
\mathrm{Pd}\left(\mathrm{Ph}_{3} \mathrm{P}\right)_{4} \\
\mathrm{rt}, 24 \mathrm{~h}\end{array}$ & $\int_{\mathrm{Ph}} \mathrm{Bu}_{3} \mathrm{Sn} \overbrace{\mathrm{SIMe}_{3}}^{16}$ & 73 \\
\hline 7. & 34 & $\begin{array}{l}\text { (a) } \mathrm{Me}_{3} \mathrm{Si}-\mathrm{SnBu}_{3} \\
\mathrm{Pd}\left(\mathrm{Ph}_{3} \mathrm{P}\right)_{4} \\
\mathrm{rt}, 24 \mathrm{~h} \\
\text { (b) } \mathrm{I}_{2}, \mathrm{CH}_{2} \mathrm{Cl}_{2}, 0{ }^{\circ} \mathrm{C}\end{array}$ & $35 \mathrm{X}=\mathrm{Bu}_{3} \mathrm{Sn}$ & $\begin{array}{l}\text { (a) } 65 \\
\text { (b) } 93 \text { (Sn-I } \\
\text { exchange) }\end{array}$ \\
\hline 8. & $\begin{array}{l}\text { 17a 1,3-cis } \\
17 \mathrm{~b} 1,3 \text {-trans }\end{array}$ & $\begin{array}{l}\mathrm{Me}_{3} \mathrm{SiSnBu}_{3} \\
\mathrm{Pd}\left(\mathrm{PPh}_{3}\right)_{4} \text { (cat.) } \\
\mathrm{C}_{6} \mathrm{H}_{6}, 70{ }^{\circ} \mathrm{C} \text { or } 25{ }^{\circ} \mathrm{C}\end{array}$ & [Si] & $\begin{array}{l}\mathbf{1 8 a} \\
(41) \\
\mathbf{1 8 b} \\
(92)\end{array}$ \\
\hline
\end{tabular}




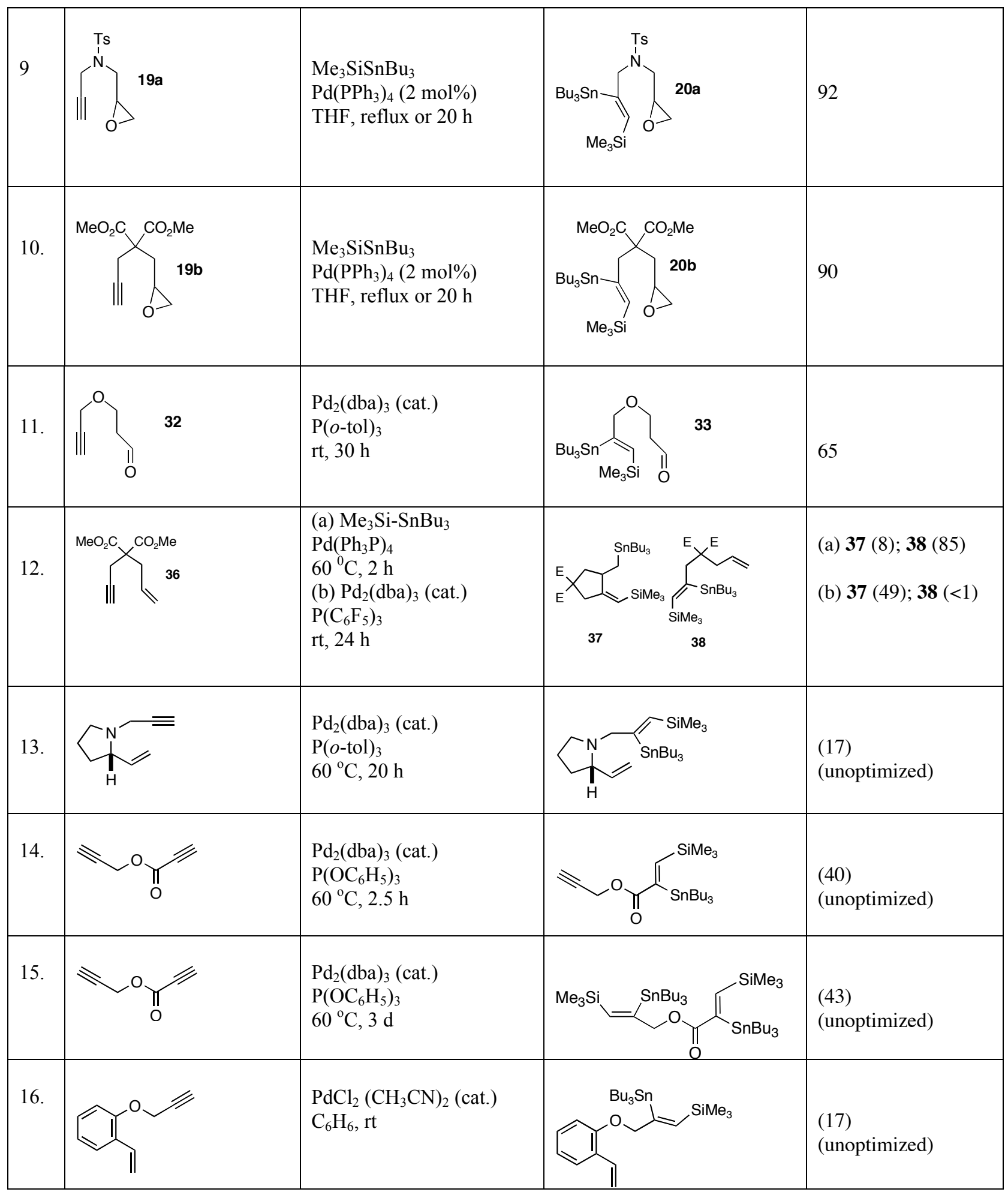




\section{${ }^{1} \mathrm{H},{ }^{13} \mathrm{C},{ }^{119}$ Sn NMR Spectra and Gas Chromatograms of Selected Compounds}

